\title{
Terahertz Plasmon Resonances in Two-Dimensional Electron Systems: Modeling Approaches
}

\author{
S. Siaber, ${ }^{1}$ S. Zonetti, ${ }^{1}$ J.E. Cunningham, ${ }^{2}$ and O. Sydoruk ${ }^{1, *}$ \\ ${ }^{1}$ Optical and Semiconductor Devices Group, Department of Electrical and Electronic Engineering, Imperial \\ College London, South Kensington Campus, London, SW7 2AZ, United Kingdom \\ ${ }^{2}$ School of Electronic and Electrical Engineering, University of Leeds, Woodhouse Lane, Leeds, LS2 9JT, \\ United Kingdom
}

(Received 17 September 2018; revised manuscript received 29 January 2019; published 27 June 2019)

\begin{abstract}
Known approaches to modeling terahertz plasmons in two-dimensional electron systems may differ significantly in their assumptions. There has, however, been little effort to analyze the differences between and application of different models to the same sets of structures. This paper discusses, develops, and compares several different theoretical approaches - namely, an effective-medium approximation, a transmission-line model, modal analysis, and full-wave simulations. In particular, we present a transmission-line model that takes into account the dielectric-air surrounding a two-dimensional system. Using modal analysis, we also solve analytically the problem of plasmon reflection and transmission when plasmons are incident on a junction between gated and ungated two-dimensional waveguides. Comparing the predictions made by the models for several structures, we find good agreement between full-wave simulations and both analytical and numerical modal analysis. The results of the effective-medium approximation and the transmissionline model also agree with each other, but differ quantitatively from those of the full-wave simulations and modal analysis. We attribute the differences to the phases of the plasmon reflection and transmission coefficients obtained with the different approaches. Our analytical expressions for the plasmon transmission and reflection coefficients represent a simple, yet accurate way to model plasmons in two-dimensional systems comprising both gated and ungated sections.
\end{abstract}

DOI: 10.1103/PhysRevApplied.11.064067

\section{INTRODUCTION}

Plasmons in two-dimensional electron systems (2DESs) hold promise for a number of terahertz devices, notably emitters [1-5] and detectors [6-9]. Traditionally, 2DESs have been realized in III-V semiconductor heterostructures [10], but recently graphene [11] has attracted increased attention.

The common geometry, for both the emitters and the detectors, derives from the field-effect transistor; see Fig. 1. A 2DES is confined between two highly conducting Ohmic contacts, which may be used to apply or detect de voltages and to connect the 2DES to antennas [12,13] and waveguides [14-16]. One or multiple gates are also usually formed above the two-dimensional (2D) channel. Application of a dc voltage between a gate and the channel changes the carrier density underneath the gate, which affects the plasmons. The gates can also be connected to antennas [17] or can form a grating [18-21] for coupling with the free-space radiation.

The Ohmic contacts and the boundaries between the gated and the ungated sections of a 2DES act as plasmon

\footnotetext{
*osydoruk@imperial.ac.uk
}

reflectors, so the whole system can then be seen as an ensemble of coupled plasmonic resonators. The geometry in Fig. 1, for example, contains three such interacting resonators formed by the gated section and the two ungated sections of the 2DES. As a result of this interaction, the system may support a spectrum of hybridized eigenmodes that cannot be reduced to either purely gated or purely ungated resonances [22].

Considerable theoretical effort has been devoted to modeling the eigenmode spectra of plasmons in 2DESs. The most popular has been the approach dating back to the pioneering work of Dyakonov and Shur [1]. The electron dynamics in a $2 \mathrm{DES}$ is described by the Euler equation, leading to a Drude expression for the 2DES conductivity. The electrostatic approximation is commonly used, so the only field quantity is the electric potential. An expression for the potential with variations in both the $x$ direction and the $z$ direction (see Fig. 1) is used to derive the plasmon dispersion relations. However, plasmon reflection and transmission are then treated by considering the boundary conditions only at the 2DES. For example, Dyakonov and Shur [1] postulated the boundary conditions of zero potential at the source and zero alternating current at the drain of a field-effect transistor. Examples of the boundary 


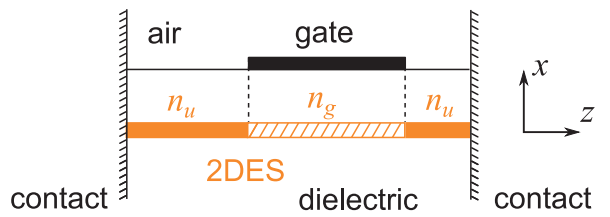

FIG. 1. A structure supporting coupled plasmons consists of three 2DES sections. The gate controls the electron density in the underlying section. Plasmons reflect from the contacts and from the junctions between the gated and the ungated 2DES.

conditions at the junctions of gated and ungated 2DESs include the ballistic current condition [23] and continuity of the potential [24]. These single-point boundary conditions may also be combined with the conditions for the whole junction; an example is the continuity of the plasmon power flow [24]. This approach often permits the analysis to be performed analytically. However, the effects of the approximations, in particular the reduction of the boundaries to a single point, often remain unclear.

Full-wave simulations are an alternative approach [25-28], in which Maxwell's equations are solved in a discretized domain, and they often prescribe the boundary conditions. Compared with analytical calculations, however, numerical simulations take more time to set up (especially, for bespoke solvers) and perform, and they may have less capacity to offer physical insights and generalizations.

Other approaches to analyze plasmons combine the rigor of solving full Maxwell's equations with the power of analytical calculations. One of them, developed by Popov et al. [29,30], assumes a plane wave incident on a structure, expands the electromagnetic fields in the Fourier integrals, and substitutes them into Maxwell's equations. The resulting integral equation is then solved numerically. Another approach, used to study plasmon reflection and transmission at waveguide junctions, is modal analysis, in which the electromagnetic fields at both sides of a junction are expanded into the waveguide eigenmodes [5,16,22,31-34]. Another example is the Wiener-Hopf technique [35,36], which has been used recently to derive expressions for plasmon transmission and reflection at a junction between two ungated 2DESs [37]. However, no analytical model has been developed so far for the practically significant scenario of plasmons incident on junctions between gated and ungated 2DESs.

In addition, there has been relatively little effort in aligning individual theoretical approaches with each other and comparing their predictions for the same configurations, which has so far largely prevented understanding the limits of applicability of various models. As a result, adoption of various theoretical approaches may also have been impeded, and the dispersion relation and the assumption of multiple half-wavelength resonances often remain the tools chosen for the analysis of experiments.
This paper discusses, develops, and compares five different approaches: effective-medium modeling, transmission-line modeling, numerical and analytical modal analysis, and full-wave simulations. Section II reviews the different approaches and expands the transmission-line model beyond its original formulation [24]. Section III discusses analytical expressions produced by different models. For the first time, the problem of plasmon incidence on a junction between a gated and an ungated waveguide is treated analytically starting with full Maxwell's equations and using modal analysis. Section IV compares all approaches for plasmon transmission and reflection at a single junction, and Sec. V compares results for plasmonic resonators comprising multiple sections of gated and ungated 2DESs. The results are discussed and conclusions are drawn in Sec. VI.

\section{MODELING APPROACHES}

Figure 1 shows the simplest geometry. The 2DES lies in the $x=0$ plane and is assumed to be infinitely long in the $y$ direction. It is confined between two Ohmic contacts, thus forming a conducting 2D channel. We assume the contacts to be infinite perfect electric conductors. A homogeneous dielectric with relative permittivity $\varepsilon$ stretches to infinity below the 2DES and at distance $d$ above it. Air fills the space $x>d$. A thin perfectly conducting gate, formed at the air-dielectric interface, divides the structure into three sections: the gated one underneath the gate and two ungated ones at both sides of the gate. The gate controls the electron density $n_{g}$ in the gated section. The densities $n_{u}$ in the ungated sections are the same.

All models we use describe the electron dynamics in the 2DES using Euler's equation in the form

$$
\frac{\partial v}{\partial t}=\frac{e}{m^{*}} E_{z}(x=0, z, t),
$$

where $v$ is the ac electron velocity, $e$ and $m^{*}$ are the electron charge and effective mass, and $E_{z}$ is the $z$ component of the electric field.

The transmission-line model developed by Aizin and Dyer [24] uses the electrostatic approximation, in which the potential $\phi(x, z, t)$ obeys Poisson's equation in the form

$$
\nabla^{2} \phi=\frac{n}{\varepsilon_{0}} \delta(x)
$$

where $n$ is the ac electron density and $\delta(x)$ is the $\delta$ function.

If we consider the potential only at the channel (i.e., for $x=0$ ), Euler's equation (1) and Poisson's equation (2) are reduced to telegrapher's equations separately for the gated and ungated 2DES in the form 


$$
\begin{aligned}
L_{u(g)} \frac{\partial j}{\partial t} & =-\frac{\partial \phi}{\partial z}, \\
C_{u(g)} \frac{\partial \phi}{\partial t} & =-\frac{\partial j}{\partial z},
\end{aligned}
$$

where $j=j(z, t)$ is the alternating-current density, $L$ and $C$ are the effective inductances and capacitances, and subscripts $u$ and $g$ denote ungated and gated sections, respectively. Equation (3) have wave solutions with angular frequency $\omega$ and wave number $q$. The original formulation assumed a homogeneous dielectric surrounding the 2DES [24]. Practical configurations, however, contain air-dielectric interfaces (see Fig. 1), taking which into account modifies both the coefficients in Eq. (3) for the ungated 2DES and the boundary conditions at junctions between the gated and ungated sections. The resulting expressions for the effective inductances and capacitances are $L_{u(g)}=m^{*} / e^{2} n_{u(g)}, C_{g}=\varepsilon_{0} \varepsilon q(1+\operatorname{coth} q d)$, and $C_{u}=2 \varepsilon_{0} \varepsilon q /(1+\Lambda)$, where $\varepsilon_{0}$ and $\varepsilon$ are vacuum and dielectric permittivities, respectively, and $\Lambda=$ $[(1-1 / \varepsilon) /(1+1 / \varepsilon)] e^{-2 q d}$.

Following Ref. [24], we impose boundary conditions at a junction between a gated and an ungated 2DES using the continuity of the potential and of the power flow. Defining $j(x, t)=I$ and $\phi(x=0, z, t)=V$, we can write the expression for the plasmon power in the form

$$
P_{u(g)}(x)=\frac{V I^{*}}{2} \xi_{u(g)},
$$

where

$$
\begin{aligned}
& \xi_{g}=1-\frac{1-e^{-2 q d}-2 q d e^{-2 q d}}{2\left(1-e^{-2 q d}\right)}, \\
& \xi_{u}=1-\frac{1+[2 q d+(3 \varepsilon+1) /(\varepsilon+1)] \Lambda}{2(1+\Lambda)},
\end{aligned}
$$

and $\lambda=(1-1 / \varepsilon) /(1+1 / \varepsilon)$. If the air-dielectric boundary is ignored $(\lambda=0, d \rightarrow \infty), \xi_{u}$ in Eq. (5) reduces to $\xi_{u} \rightarrow \frac{1}{2}$, which is the original result in Ref. [24]. We then define effective impedances as

$$
\tilde{Z}_{u(g)}=\frac{V}{I \xi_{u(g)}}=\frac{1}{\xi_{u(g)}} \sqrt{\frac{L}{C}}=\frac{q}{\omega C_{u(g)} \xi_{u(g)}}
$$

so that

$$
\begin{aligned}
& \tilde{Z}_{g}=\frac{1}{\varepsilon_{0} \varepsilon \omega(1+\operatorname{coth} q d)}\left(\frac{1}{2}+\frac{q d e^{-2 q d}}{1-e^{-2 q d}}\right)^{-1}, \\
& \tilde{Z}_{u}=\frac{(1+\Lambda)}{2 \varepsilon_{0} \varepsilon \omega}\left(1-\frac{1+\left(2 q d+\frac{3 \varepsilon+1}{\varepsilon+1}\right) \Lambda}{2(1+\Lambda)}\right)^{-1} .
\end{aligned}
$$

The voltages and currents at the ends of a section of a transmission line are readily obtained with use of the transmission matrix [38] in the form

$$
\left(\begin{array}{c}
V_{i} \\
I_{i}
\end{array}\right)=\left(\begin{array}{cc}
\cos q_{\alpha} L_{\alpha} & i Z_{\alpha} \sin q_{\alpha} L_{\alpha} \\
i \\
Z_{\alpha} \sin q_{\alpha} L_{\alpha} & \cos q_{\alpha} L_{\alpha}
\end{array}\right)\left(\begin{array}{c}
V_{j} \\
I_{j}
\end{array}\right)
$$

where $L_{\alpha}$ is the length of a section, $Z_{\alpha}$ is its impedance, and $q_{\alpha}$ is the wave number. A system comprising multiple sections can be characterized by a total transmission matrix $\hat{T}$, which is a product of the transmission matrices all elements. The spectrum of eigenfrequencies can be found by equating the element $(1,2)$ of the transmission matrix to zero.

\section{A. Modal analysis}

Modal analysis originated in the theory of closed metallic waveguides (see, e.g., Ref. [39]), and was then adopted to open waveguides [40], including three-dimensional plasmonic ones [41]. It has been also recently used to describe plasmon reflection and transmission of $2 \mathrm{D}$ plasmons [31-34].

The analysis of a 2D plasmonic waveguide starts by assuming transverse magnetic waves $\left(E_{x}, E_{z}\right.$, and $H_{y}$ nonzero components of the fields). Substituting them into Maxwell's equations and using the standard field boundary conditions yields eigenmode spectra of gated and ungated waveguides, which can be separated into two parts for modes with discrete and continuous wave numbers. The discrete part corresponds to plasmons, and the continuous part corresponds to radiation and evanescent modes. The plasmons obey dispersion relations of the form

$$
\Gamma_{g}+\left(1-\Gamma_{g}\right) e^{-2 i k_{x d} d}=0
$$

for a waveguide formed by a gated 2DES and

$$
\left(1-\Gamma_{u}\right)(1+\zeta) e^{-2 i k_{x d} d}=\Gamma_{u}(1-\zeta)
$$

for a waveguide formed by an ungated 2DES. Here $k_{x, p}^{u(g)}$ are the plasmon wave numbers in ungated and gated waveguides respectively, $\Gamma_{u(g)}=\gamma_{u(g)}\left(k_{x, p}^{u(g)} / \omega^{2}\right)=$ $-\left(i e^{2} n_{u(g)} / 2 m^{*} \varepsilon \varepsilon_{0}\right)\left(k_{x, p}^{u(g)} / \omega^{2}\right)$, and $\zeta=k_{x, p}^{u} / \varepsilon k_{x, p}^{u, \text { air }}$.

A plasmon incident on a junction between a gated and an ungated waveguide will partially reflect from and partially transmit through the junction. Because of the different plasmon field profiles in the two waveguides, the incident plasmon will also excite modes from the continuous part of the spectrum.

The boundary conditions for the plasmon incidence from the ungated section (continuity of $x$ component of the electric field and the $y$ component of the magnetic field) 
can then be written in the following forms:

$$
\begin{aligned}
H_{y, u}^{(1)} & +R_{\mathrm{ug}} H_{y, u}^{(1)}+\int_{0}^{\infty} r_{u t}\left(k_{x}^{e}\right) h_{y, u t}^{(1)} d k_{x}^{e} \\
& +\int_{0}^{\infty} r_{u b}\left(k_{x}^{e}\right) h_{y, u b}^{(1)} d k_{x}^{e}=\int_{0}^{\infty} t_{g t}\left(k_{x}^{e}\right) h_{y, g t}^{(1)} d k_{x}^{e}
\end{aligned}
$$

for continuity of the $x$ component of the electric field in air,

$$
\begin{aligned}
& H_{y, u}^{(2,3)}+R_{\mathrm{ug}} H_{y, u}^{(2,3)}+\int_{0}^{\infty} r_{u t}\left(k_{x}^{e}\right) h_{y, u t}^{(2,3)} d k_{x}^{e} \\
& +\int_{0}^{\infty} r_{u b}\left(k_{x}^{e}\right) h_{y, u b}^{(2,3)} d k_{x}^{e}=T_{\mathrm{ug}} H_{y, g}^{(2,3)} \\
& +\int_{0}^{\infty} t_{g b}\left(k_{x}^{e}\right) h_{y, g b}^{(2,3)} d k_{x}^{e}
\end{aligned}
$$

for continuity of the $x$ component of the electric field in the dielectric,

$$
\begin{aligned}
& k_{z, p}^{u}\left(1-R_{\mathrm{ug}}\right) H_{y, u}^{(1)}-\int_{0}^{\infty} k_{z, e}^{u} r_{u t}\left(k_{x}^{e}\right) h_{y, u t}^{(1)} d k_{x}^{e} \\
& \quad-\int_{0}^{\infty} k_{z, e}^{u} r_{u b}\left(k_{x}^{e}\right) h_{y, u b}^{(1)} d k_{x}^{e}=\int_{0}^{\infty} k_{z, e}^{g} t_{g t}\left(k_{x}^{e}\right) h_{y, g t}^{(1)} d k_{x}^{e}
\end{aligned}
$$

for continuity of the $y$ component of the magnetic field in air, and

$$
\begin{aligned}
& k_{z, p}^{u}\left(1-R_{\mathrm{ug}}\right) H_{y, u}^{(2,3)}-\int_{0}^{\infty} k_{z, e}^{u} r_{u t}\left(k_{x}^{e}\right) h_{y, u t}^{(2,3)} d k_{x}^{e} \\
& -\int_{0}^{\infty} k_{z, e}^{u} r_{u b}\left(k_{x}^{e}\right) h_{y, u b}^{(2,3)} d k_{x}^{e}=k_{z, p}^{g} T_{\mathrm{ug}} H_{y, g}^{(2,3)} \\
& +\int_{0}^{\infty} k_{z, e}^{g} t_{g b}\left(k_{x}^{e}\right) h_{y, g b}^{(2,3)} d k_{x}^{e}
\end{aligned}
$$

for continuity of the $y$ component of the magnetic field in the dielectric. In Eqs. (12a)-(12d), $H_{y}^{(1)}$ and $h_{y}^{(1)}$ denote magnetic fields of the plasmon and the radiation and evanescent modes in air (the subscript $p$ denotes plasmons and the subscript $e$ denotes the radiation and evanescent modes), while $H_{y}^{(2,3)}$ and $h_{y}^{(2,3)}$ are the components in the dielectric above and below the 2DES. The plasmon reflection and transmission coefficients are $R$ and $T$, and the reflection and transmission coefficients of the modes of the continuous spectrum are $r$ and $t$. The superscripts $t$ and $b$ correspond to the "top" and "bottom" modes of the continuous spectrum [33]. The subscript ug denotes incidence from the ungated waveguide and the subscript gu denotes incidence from the gated waveguide. In the next step, the dependence on the $x$ coordinate in Eqs. (12a)-(12d) is eliminated with use of the mode orthogonality conditions, so Eqs. (12a)-(12d) transform into a system of integral-algebraic equations for the reflection and transmission coefficients of plasmons and of modes from the continuous parts of the spectrum. These modal equations can then be solved numerically.

\section{B. Full-wave simulations}

Finite-element analysis (FEA) is an established numerical method, which is particularly effective in simulating systems that can be reduced to two spatial dimensions, as is the case in this work. Our package of choice is the commercial software program COMSOL MULTIPHYSICS, which provides dedicated tools for FEA of full-wave electromagnetics alongside JAVA coding capabilities. When calculating the plasmon transmission and reflection coefficients for a single junction, we model a $2 \mathrm{DES}$ as a 1-nm-thick layer with a Drude permittivity, and simulate the plasmon field profiles using boundary-mode analysis. Afterwards, plasmons are launched and detected at two ports, which allows us to calculate the corresponding $S$ parameters. To avoid nonplasmonic modes, which are excited at the junction, affecting the $S$ parameters, the gated and ungated waveguides have to be tens of micrometers long. When calculating the resonant frequencies, we model a 2DES using the surface-current boundary condition. The vertical extent of the modeling domain is necessarily finite in a FEA approach. Because our interest is in plasmons, whose fields decay exponentially away from the 2DES, we find it sufficient to have a domain with a height of $100 \mu \mathrm{m}$ and to terminate it, at the top and bottom, by perfect magnetic conductors. Ohmic contacts and gates are taken to be perfect electric conductors.

\section{ANALYTICAL SOLUTIONS}

There are several theoretical approaches to the problem of plasmon transmission and reflection that yield results in an analytical form.

Perhaps the simplest approach is an effective-medium approximation, in which the gated and ungated waveguides are represented as homogeneous media with different plasmon velocities. After finding the wave numbers in each waveguide by solving the plasmon dispersion relations (10) and (11), we can readily obtain the plasmon reflection and transmission coefficients from Fresnel's equations as

$$
\begin{gathered}
R_{\mathrm{ug}}=\frac{k_{u}-k_{g}}{k_{u}+k_{g}}, \quad T_{\mathrm{ug}}=\frac{2 k_{u}}{k_{u}+k_{g}}, \\
R_{\mathrm{gu}}=\frac{k_{g}-k_{u}}{k_{u}+k_{g}}, \quad T_{\mathrm{gu}}=\frac{2 k_{g}}{k_{u}+k_{g}} .
\end{gathered}
$$

The effective-medium approximation does not take into account the spatial configuration of the plasmon fields. 
Thus, junction geometry affects only plasmon wave numbers in the two waveguides, but does not explicitly influence the expressions for the transmission and reflection coefficients. Therefore, the expressions in Eq. (13) remain the same for junctions between two gated or two ungated waveguides.

The transmission-line model offers a similar set of expressions for the transmission and reflection coefficients, this time based on the waveguide impedances, $Z_{u(g)}$, in the form

$$
\begin{gathered}
R_{\mathrm{ug}}=\frac{Z_{g}-Z_{u}}{Z_{g}+Z_{u}}, \quad T_{\mathrm{ug}}=\frac{2 Z_{g}}{Z_{g}+Z_{u}}, \\
R_{\mathrm{gu}}=\frac{Z_{u}-Z_{g}}{Z_{g}+Z_{u}}, \quad T_{\mathrm{gu}}=\frac{2 Z_{u}}{Z_{g}+Z_{u}} .
\end{gathered}
$$

Another analytical approximation for the reflection and transmission coefficients was obtained in Ref. [33] for the special case of two ungated 2DES embedded in a homogeneous dielectric with close values of the electron density. Expressions for two ungated graphene plasmon waveguides with arbitrary values of electron density were presented in Ref. [37].

In the absence of loss, $k_{u(g)}$ and $Z_{u(g)}$ are real, leading to real-valued reflection and transmission coefficients in Eqs. (13) and (14). However, previous calculations using modal analysis showed that the coefficients may also have a nonzero phase $[33,34]$. The presence of a nonzero phase was also reported in Ref. [37] for two ungated waveguides. However, no analytical expressions taking the phase into account appear to have been reported for the practically significant case of the junction between a gated and an ungated waveguide.

Our starting point for deriving such expressions is Eqs. (12a)-(12d), representing the boundary conditions at a junction between a gated and an ungated waveguide. We then assume a plasmon incident from the ungated waveguide and use the variational method. To do so, we take the magnetic field at the boundary, $\mathcal{H}_{y}$, as being proportional to the field of the incident plasmon only, that is,

$$
\mathcal{H}_{y}=C_{u} H_{u}
$$

where $C_{u}$ is a variational parameter.
Substituting Eq. (15) into Eqs. (12a)-(12d) yields an expression for $C_{u}$ in the form

$$
C_{u}\left(k_{z, p}^{u} S_{p}^{u}+k_{z, p}^{g} \frac{J_{p p}^{2}}{S_{p}^{g}}+\int_{0}^{\infty} k_{z} \frac{J_{p t}^{2}}{S_{t}^{g}} d k_{x}^{e}+\int_{0}^{\infty} k_{z} \frac{J_{p b}^{2}}{S_{b}^{g}} d k_{x}^{e}\right)=2,
$$

where the overlap integrals for the plasmons and for the evanescent modes are defined as

$$
\begin{aligned}
S_{p}^{u(g)}= & \int_{d}^{\infty}\left(H_{y ; u(g)}^{(1)}\right)^{2} d x \\
& +\frac{1}{\varepsilon}\left[\int_{0}^{d}\left(H_{y ; u(g)}^{(2)}\right)^{2} d x+\int_{-\infty}^{0}\left(H_{y ; u(g)}^{(3)}\right)^{2} d x\right] \\
S_{t, b}^{g}= & \int_{d}^{\infty}\left(h_{y ; g t g b}^{(1)}\right)^{2} d x \\
& +\frac{1}{\varepsilon}\left[\int_{0}^{d}\left(h_{y ; g t, g b}^{(2)}\right)^{2} d x+\int_{-\infty}^{0}\left(h_{y ; g t, g b}^{(3)}\right)^{2} d x\right] .
\end{aligned}
$$

The following overlap integrals are also defined. The plasmon overlap integral is of the form $J_{p p}=J_{p, g}^{p, u}=\int_{d}^{\infty}$ $H_{y, u}^{(1)} H_{y, g}^{(1)} d x+(1 / \varepsilon)\left(\int_{0}^{d} H_{y, u}^{(2)} H_{y, g}^{(2)} d x+\int_{-\infty}^{0} H_{y, u}^{(3)} H_{y, g}^{(3)} d x\right)$. The overlap integrals of the ungated plasmon and evanescent gated modes are of the form $J_{p t}=J_{p, u}^{e, g}=\int_{d}^{\infty} H_{y, u}^{(1)}$ $h_{y ; g t}^{(1)} d x+(1 / \varepsilon)\left(\int_{0}^{d} H_{y, u}^{(2)} h_{y ; g t}^{(2)} d x+\int_{-\infty}^{0} H_{y, u}^{(3)} h_{y ; g t}^{(3)} d x\right) \quad$ and $J_{p b}=J_{p, u}^{e, g}=\int_{d}^{\infty} H_{y, u}^{(1)} h_{y ; g b}^{(1)} d x+(1 / \varepsilon) \quad\left(\int_{0}^{d} H_{y, u}^{(2)} h_{y ; g b}^{(2)} d x+\right.$ $\left.\int_{-\infty}^{0} H_{y, u}^{(3)} h_{y ; g b}^{(3)} d x\right)$, where $J_{p t}$ is the overlap integral between the plasmon and the modes over the gate (top modes) and $J_{p b}$ is the overlap integral between the plasmon and the modes under the gate (bottom modes).

These expressions can be simplified by noticing that the overlap between the plasmon and the modes below the gate is likely to be greater than the overlap between the plasmon and the modes above the gate: $\left|J_{p t}^{2} / S_{t}^{g}\right| \ll$ $\left|J_{p} b^{2} / S_{b}^{g}\right|$ for all $k_{x}^{e}$. This leads to (see Appendix B for details)

$$
C_{u}=\frac{\varepsilon}{k_{z, p}^{u} / 4 \kappa_{u}+\frac{\frac{k_{z, p}^{g}}{\kappa g}\left(1+\frac{\kappa g}{\kappa_{g}+\kappa_{u}}\right)^{2}}{\left(1+\operatorname{coth} \kappa_{g} d\right)\left[1+\kappa_{g} d\left(\operatorname{coth} \kappa_{g} d-1\right)\right]}+\frac{\kappa_{u}^{2}}{\pi} \int_{0}^{k_{\mathrm{co}}} \sqrt{\varepsilon \frac{\omega^{2}}{c^{2}}-k^{2}} \frac{d k}{\left(\kappa_{u}^{2}+k^{2}\right)^{2}\left(1-\frac{4 k^{4} \gamma_{g}^{2} d^{2}}{\omega^{4}}\right)}},
$$


where $\kappa_{u(g)}=i k_{x, p}^{u(g)} \geq 0$ and the cutoff wave number $k_{\mathrm{co}}=$ $1 / 2 d$. The plasmon reflection and transmission coefficients are then found as

$$
R_{\text {ug }}=C_{u}-1, \quad T_{\text {ug }}=\frac{J_{p p}}{S_{p}^{g}} C_{u} .
$$

The reflection and transmission coefficients for a plasmon incident from the opposite direction (from the gated waveguide) can be found, using reciprocity relations [42], as

$$
\begin{aligned}
& T_{\mathrm{gu}}=\frac{k_{z, p}^{g} S_{p}^{g}}{k_{z, p}^{u} S_{p}^{u}} T_{\mathrm{ug}}=\frac{k_{z, p}^{g} J_{p p}}{k_{z, p}^{u} S_{p}^{u}} C_{u}, \\
& R_{\mathrm{ug}}=-1+\frac{J_{p p}}{S_{p}^{g}} T_{\mathrm{gu}}=-1+\frac{k_{z, p}^{g} J_{p p}^{2}}{k_{z, p}^{u} S_{p}^{u} S_{p}^{g}} C_{u} .
\end{aligned}
$$

The expressions for the transmission and reflection coefficients can be simplified radically by assuming that the ungated density, $n_{u}$, is comparable to or larger than the gated density, $n_{g}$ (see Appendix B), leading to

$$
\begin{gathered}
R_{\mathrm{ug}}=\frac{1-2 \kappa_{\mathrm{g}} d+i \frac{2}{\pi}}{1+2 \kappa_{\mathrm{g}} d-i \frac{2}{\pi}}, \quad T_{\mathrm{ug}}=\frac{2 \kappa_{g} d}{1+2 \kappa_{g} d-i \frac{2}{\pi}}, \\
R_{\mathrm{gu}}=\frac{-1+2 \kappa_{g} d+i \frac{2}{\pi}}{1+2 \kappa_{g} d-i \frac{2}{\pi}}, \quad T_{\mathrm{gu}}=\frac{4}{1+2 \kappa_{g} d-i \frac{2}{\pi}} .
\end{gathered}
$$

The expressions in Eqs. (21a) and (21b) conserve power, so $\left|R_{\alpha \beta}\right|^{2}+\kappa_{\beta} S_{p}^{\beta} / \kappa_{\alpha} S_{p}^{\alpha}\left|T_{\alpha \beta}\right|^{2}=1$, where $\alpha, \beta$ is $u$ or $g$, and $S_{p}^{g}=1 / \kappa_{g}^{2} d, S_{p}^{u}=1 / 2 \kappa_{u}$.

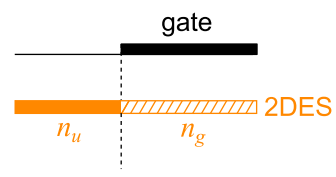

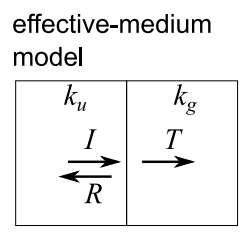

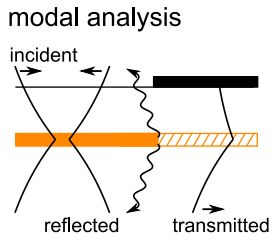

transmission-line model

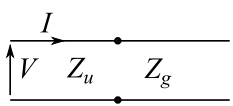

full-wave simulations

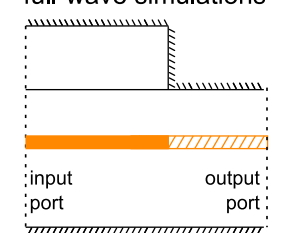

FIG. 2. Plasmon transmission and reflection at a junction between a gated and an ungated waveguide is analyzed by different methods.

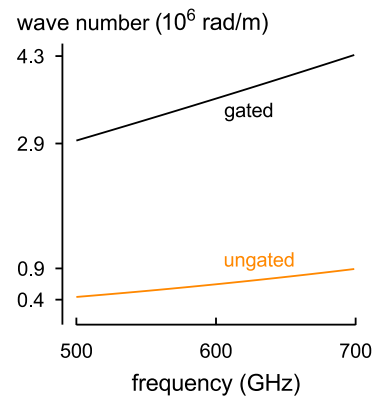

FIG. 3. Dispersion curves for gated and ungated plasmons. The ungated electron density is $6.5 \times 10^{11} \mathrm{~cm}^{-2}$; the gated electron density is $4.9 \times 10^{11} \mathrm{~cm}^{-2}$.

\section{PLASMON REFLECTION AND TRANSMISSION AT A JUNCTION}

This section compares the results of different models (see Fig. 2) for the plasmon reflection and transmission coefficients at a single junction between an ungated and a gated waveguide. For the numerical examples, we take the parameters of the Ga-As/Al-Ga-As heterostructure from the recent experiments by Wu et al. $[15,16]$ The thickness $d$ of the dielectric above the $2 \mathrm{DES}$ is $75 \mathrm{~nm}$. The relative permittivity $\varepsilon$ of the dielectric is 12.4. The ungated electron density $n_{u}$ is $6.5 \times 10^{11} \mathrm{~cm}^{-2}$; the gated electron density $n_{g}$ is $4.9 \times 10^{11} \mathrm{~cm}^{-2}$ (in the middle of the density range chosen). Figure 3 shows the plasmon dispersion curves for the corresponding gated and ungated 2DES in the frequency range from 500 to $700 \mathrm{GHz}$.

Figures 4(a) and 4(b) show, respectively, the amplitude and the phase of the reflection coefficient assuming a plasmon incident from the ungated waveguide. Figures 4(c) and 4(d) show the same assuming incidence from the gated waveguide. Subfigures in the figures have the same range of frequency. In these subfigures some ticks labels and captions are omitted as being redundant. The curves are calculated by different methods: the effective-medium model, the transmission-line model, the numerical and analytical solutions of the mode-matching equations, and the full-wave simulations. (Because the effective-medium and transmission-line models can yield only real-valued coefficients, their phases are not shown.)

According to all models, the absolute values of the reflection coefficient [see Figs. 4(a) and 4(c)] decrease with increasing frequency. The numerical solutions of the modal equations agree well with the full-wave simulations. The phases of the reflection coefficients calculated by three models [see Figs. 4(b) and 4(d)] also decrease with increasing frequency. The phase for plasmon incidence from the ungated channel is around $\pi / 4$ for all models. When a plasmon is incident from the gated channel, the phase is close to $7 \pi / 8$ when calculated numerically from 


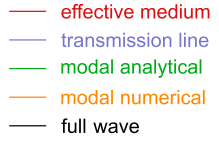

(a) $\left|R_{\mathrm{ug}}\right|$

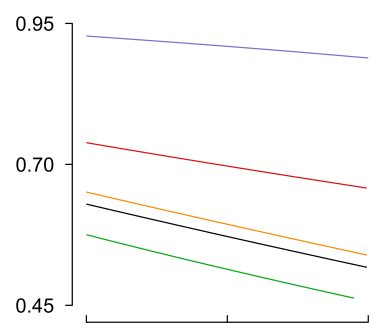

(c) $\left|R_{\text {gut }}\right|$

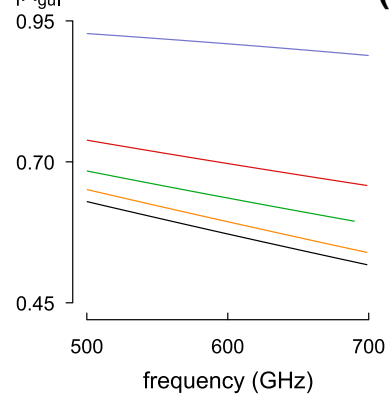

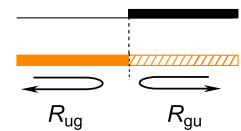

(b) phase of $R_{\mathrm{ug}}$

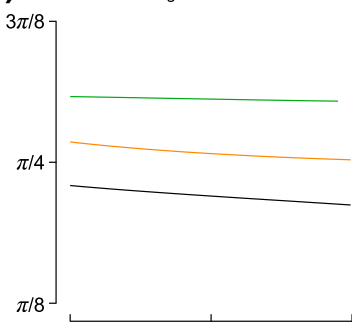

(d) phase of $R_{\mathrm{gu}}$

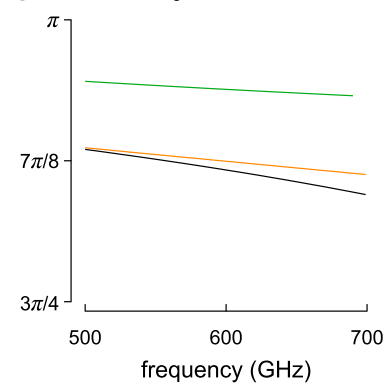

FIG. 4. Plasmon reflection coefficients at a junction between a gated and an ungated 2DES calculated by five different models. The best agreement is between modal analysis and full-wave simulations.

the modal equations and from full-wave simulations, but it is somewhat higher when calculated analytically from the modal equations.

Comparison between the transmission coefficients obtained with different models is complicated because each model uses different quantities and normalizations. However, such a comparison can be made easily for modal analysis and full-wave simulations, which rely on Maxwell's equations. Figure 5 shows the absolute values and the phases of the transmission coefficients calculated from the full-wave simulations and the numerical and analytical solutions of the modal equations. As in previous Figure, subfigures in the figures have the same range of frequency and redundant tick labels are omitted. The absolute values of the transmission coefficients increase with frequency, and the best agreement is, as before, between the numerical solutions of the modal equations and the full-wave simulations. The phases of the transmission coefficients are below $\pi / 8$. The transmission coefficients are the same for both incidence directions when calculated by the numerical modal analysis and the full-wave simulations.

We find similar behavior of the coefficients calculated by all models for other values of the gated electron density.

\section{EIGENFREQUENCIES OF PLASMONIC RESONATORS}

This section discusses the eigenfrequency spectrum of plasmonic devices comprising alternating gated and ungated 2D channels confined between two Ohmic contacts (see Fig. 1). The transmission-line model tackles the problem using the transmission matrix; see Eq. (9). Our full-wave calculations rely on the eigenmode solver of COMSOL MULTIPHYSICS. On the other hand, when the plasmon transmission and reflection coefficients are found by the mode-matching technique [Eqs. (12a)-(12d), (19), and (20)] or from the effective-medium approximation [Eq. (13)], the eigenmodes can be found using the theory of rays [43]. Assuming that the fields of the evanescent modes decay quickly enough away from a junction, only plasmons will be able to reach the next junction. A propagating plasmon is then considered to be a ray undergoing multiple transmissions and reflections, and the total reflection and transmission are given by the sum of all paths taken by the plasmon.

We compare the models using three examples chosen to demonstrate different behavior of the resonances. In the first two examples, we concentrate on anticrossing of resonance curves due to the interaction between gated and ungated resonators. In the third example, we demonstrate splitting of the initially identical resonance curves due to

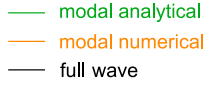

(a) $\mid$

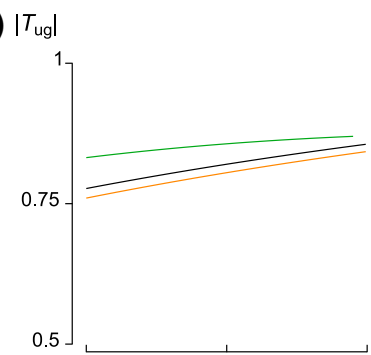

(c) $\mid T_{\mathrm{g}}$

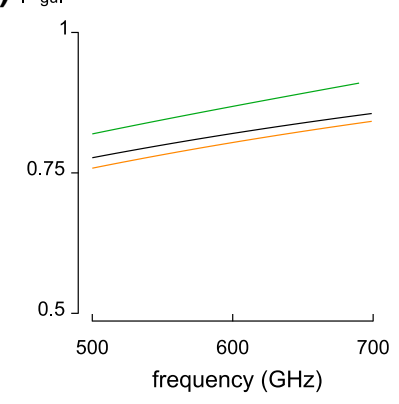

(b) phase of $T_{\mathrm{ug}}$

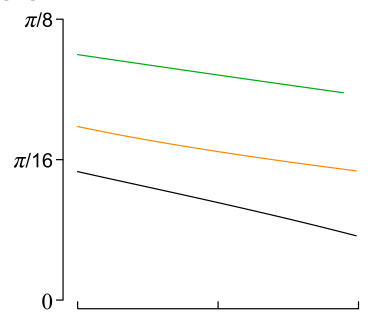

(d) phase of $T_{\mathrm{gu}}$

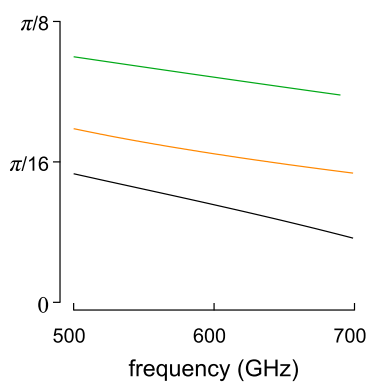

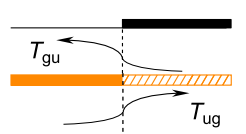

FIG. 5. Plasmon transmission coefficients at a junction between a gated and an ungated 2DES calculated by three models relying on solving Maxwell's equations directly. 
the interaction of two gated resonators coupled by sections of the ungated 2DES. The conclusions we are able to draw, however, are general and are not limited to these specific examples.

Subfigures in the figures that illustrate results obtained for different models have the same range of frequency and concentration or distance between channel and metallic gate. Similarly, in these subfigures some ticks labels and captions are omitted.

Our first device consists of three sections: a $20-\mu \mathrm{m}$ ungated section, a $3-\mu \mathrm{m}$ gated section, and a $3-\mu \mathrm{m}$ ungated section. This choice of lengths allows us to focus on interaction between plasmons in the gated section and the longer ungated section. The short ungated section does not affect the resonances, but it still has to be retained so that the gated electron density may be controlled by a dc voltage. The dc electron densities in the ungated sections, $n_{u}$, are both $6.5 \times 10^{11} \mathrm{~cm}^{-2}$. The gated electron density varies between $3.2 \times 10^{11}$ and $6.5 \times 10^{11} \mathrm{~cm}^{-2}$.

In the frequency region from 500 to $700 \mathrm{GHz}$, the isolated gated section would support three resonances accommodating three, four, and five half-wavelengths. The longer ungated section would also support three resonances with the same number of half-wavelengths, while the shorter ungated section would support no modes, being below the cutoff. These "pure" resonances are shown in Fig. 6(a) for the range of gated electron densities chosen. The ungated resonances are independent of the density and, therefore, appear as vertical lines. The gated resonances appear as slanted lines, due to their square-root dependence on the density.

The "pure" gated and ungated resonance lines cross at a number of points, and in the coupled system in Fig. 1 where the resonators interact, one would expect to see a typical anticrossing behavior. This is indeed a result of calculations by the different methods shown in Figs. 6(b)-6(f): the effective-medium model [Fig. 6(b)], the original transmission-line model ignoring the airdielectric interface [Fig. 6(c)], the modified transmissionline model [Fig. 6(d)], the numerical solution of the modematching equations [Fig. 6(e)], the analytical solutions of the mode-matching equations [Fig. 6(f)], and the full-wave simulations. To aid comparison between them, the results of the full-wave simulations (dashed lines) are plotted in all subfigures.

The anticrossing regions calculated by the effectivemedium model [see Fig. 6(b)] lie as predicted by the "pure" resonances in Fig. 6(a). However, the anticrossing regions predicted by the full-wave simulations are shifted toward higher frequencies and electron densities relative to the results obtained with the effective-medium model. The likely reason is the phase of the reflection coefficients $R_{\mathrm{ug}}$. A resonator will accommodate an integer number of wavelengths only if the product of the reflection coefficients at both ends is a real number. This is indeed the case for the effective-medium model, but is not the case for the fullwave simulations, which predict a nonzero phase for $R_{\mathrm{ug}}$ (see Fig. 4). Another difference from Fig. 6(a) is that the effective-medium model predicts slightly lower strengths of the resonance interaction (as manifested by the size of the anticrossing regions), especially at lower electron densities.

The resonance curves calculated with the original transmission-line model, shown in Fig. 6(c), differ from those obtained with the full-wave calculations, even in

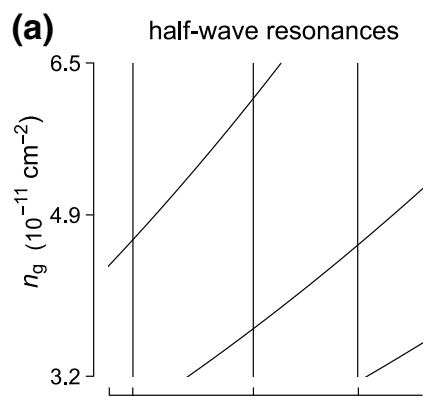

(b) effective medium
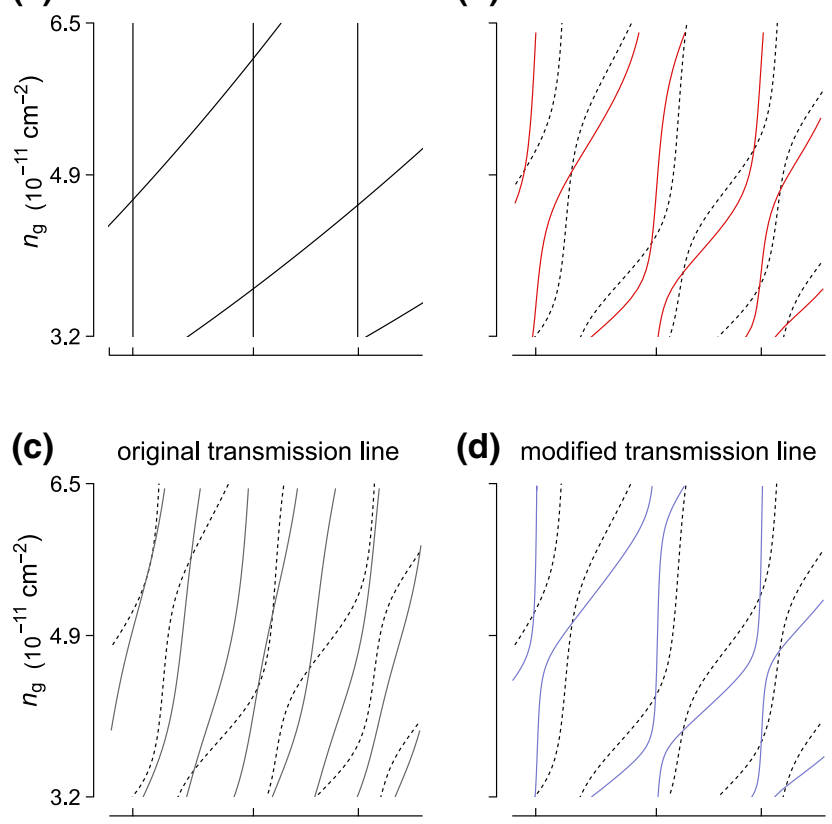

(d) modified transmission line
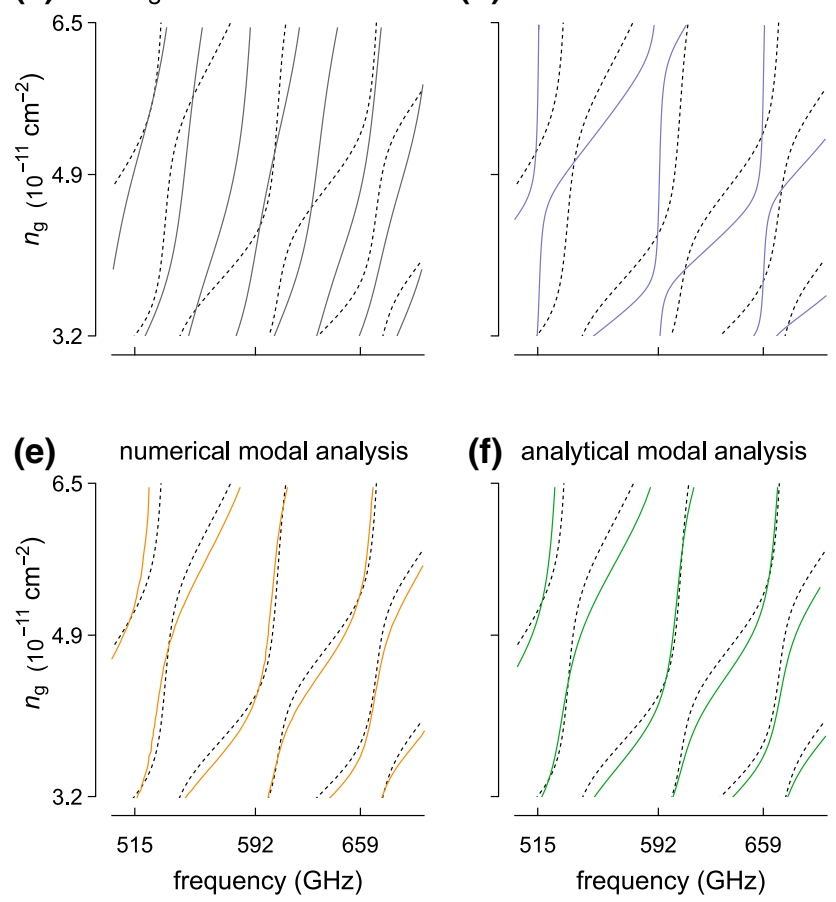

(f) analytical modal analysis

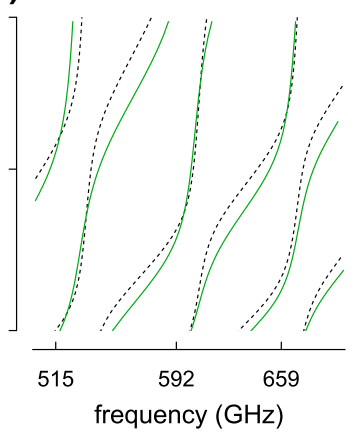

FIG. 6. Resonances of a three-section structure that has a gated section of length $3 \mu \mathrm{m}$ and two ungated sections of lengths 3 and $20 \mu \mathrm{m}$ calculated by five different models. Full-wave simulations are shown in (b)-(f) by dashed lines for comparison and as a guide for the eye. The half-wavelength resonances of the isolated sections are shown in (a). The best quantitative agreement is between the full-wave simulations and the analytical and numerical modal analysis. The modified transmission-line model and the effective-medium model also agree with each other. 


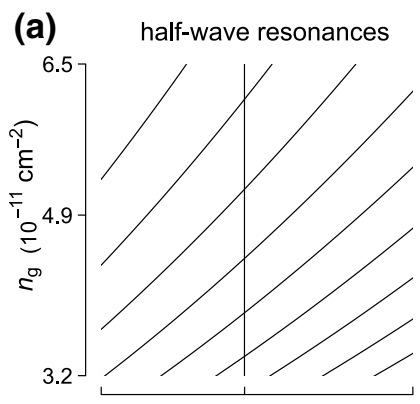

(b) effective medium

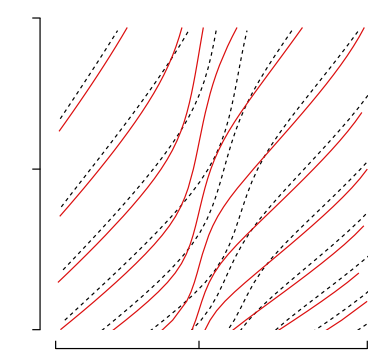

(c) original transmission line

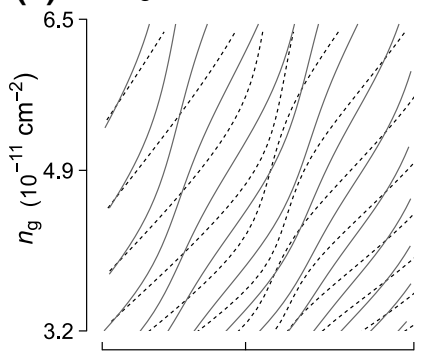

(d) modified transmission line

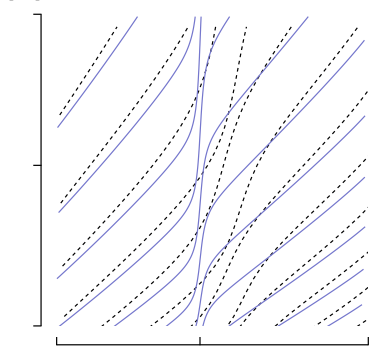

(e) numerical modal analysis

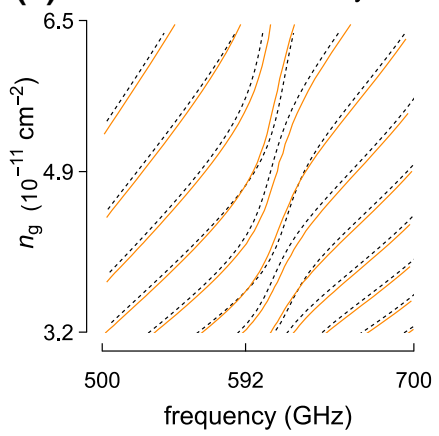

(f) analytical modal analysis

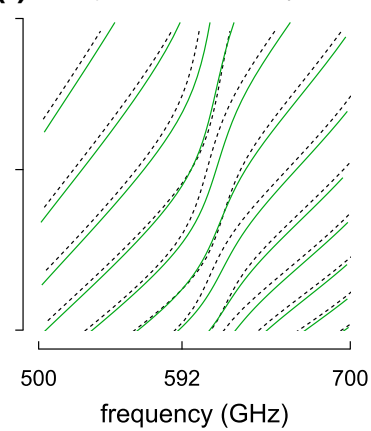

FIG. 7. Resonances of a three-section structure that has a gated section and two ungated sections each of length $10 \mu \mathrm{m}$. Full-wave simulations are shown in (b)-(f) by dashed lines for comparison and as a guide for the eye. The half-wavelength resonances of the isolated sections are shown in (a). The fullwave simulations and the modal analysis, on the one hand, and the finite-element and modified transmission-line models, on the other hand, disagree around $592 \mathrm{GHz}$, where the ungated resonances dominate, as shown in (a).

the total number of resonances. However, the qualitative agreement is restored by the modified transmissionline model; see Fig. 6(d). These results also agree with results of the effective-medium calculations; see Fig. 6(b). Both predict the same positions of the anticrossing regions (which is expected, since both models give realvalued plasmon reflection and transmission coefficients). The transmission-line model, however, predicts stronger interaction between the resonators, and agrees better with the full-wave simulations for the three highest resonances.
Of the five models, the numerical solution of the modematching equations agrees best with the full-wave simulations across the whole parameter space; see Fig. 6(e). The analytical solution of the mode-matching equations offers slightly worse agreement; see Fig. 6(f).

Our next example is a structure again with three 2DES sections, but each now having length $10 \mu \mathrm{m}$. The ungated sections are now identical, and the gated section interacts equally with both of them. The resonance curves are shown in Fig. 7 with the same presentation as before. The isolated ungated sections each support a single resonance accommodating a whole wavelength and the isolated gated section supports 11 resonances; see Fig. 7(a). A pattern similar to that in the previous example can be seen in Figs. 7(b)-7(f). The gated and ungated resonators interact strongly with each other around $590 \mathrm{GHz}$, leading to the anticrossing behavior of the resonance curves. Outside this region, the resonances can be seen as "pure" gated resonances perturbed by the ungated sections separating the gated section from the Ohmic contacts. The results obtained with the original transmission-line model disagree with the results obtained with the other models. The full-wave simulations agree with the numerical and analytical solutions of the mode-matching equations.

Outside the interaction region, all four models agree with each other. The gated resonances dominate, and their resonant frequencies are defined by reflections in the gated section. The phase of the corresponding reflection coefficients, $R_{\mathrm{gu}}$, predicted by the mode-matching and fullwave calculations [see Fig. 4(d)] is close to $\pi$, and is therefore in agreement with the transmission-line and the effective-medium models.

However, for frequencies around $590 \mathrm{GHz}$, reflections in the ungated sections influence the resonances, and the phase of the reflection coefficient $R_{\mathrm{ug}}$ becomes important. The modal analysis and full-wave simulations predict a phase value close to $\pi / 4$. The other models are unable to predict a nontrivial phase; hence, the disagreement in the results.

Our next example is a structure consisting of five sections: two gated and three ungated sections each of length $10 \mu \mathrm{m}$; see Fig. 8. The isolated resonances are the same as in the previous example. The new feature now is that the two gated resonators can interact with each other via

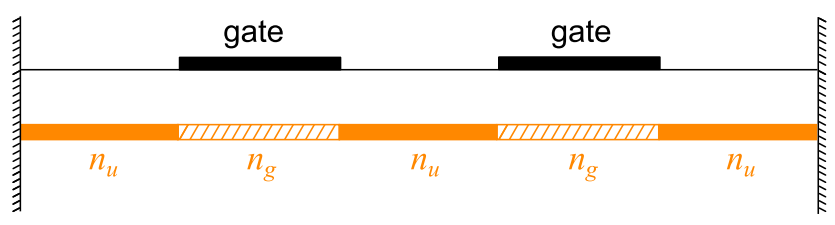

FIG. 8. Five-section structure with two gated sections. The gated electron densities are identical. All sections have the same length. 


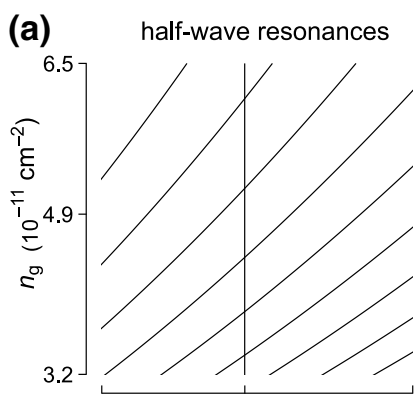

(c) original transmission line

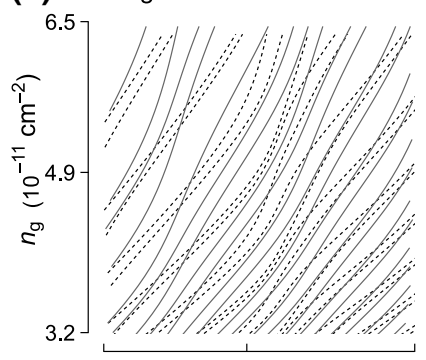

(d)

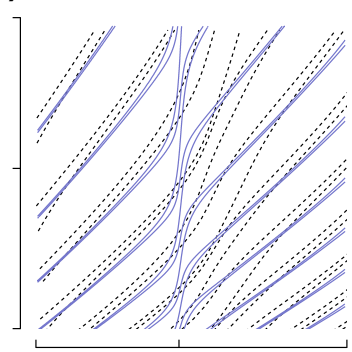

(e) numerical modal analysis

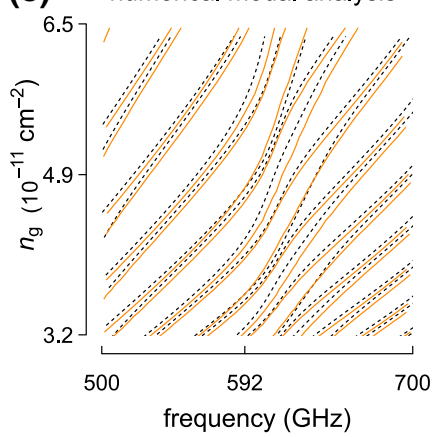

(f) analitycal modal analysis

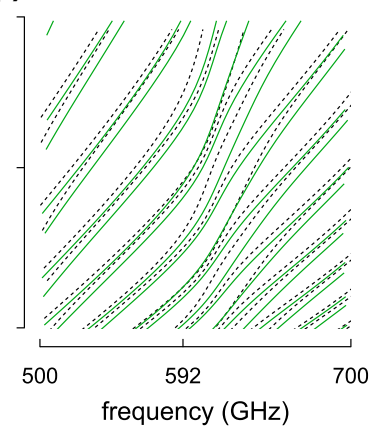

FIG. 9. Resonances of the three-section structure shown in Fig. 8, which has two gated sections and three ungated sections each of length $10 \mu \mathrm{m}$ (b)-(f). Full-wave simulations are shown in (b) (f) by dashed lines for comparison and as a guide for the eye. The half-wavelength resonances of the isolated sections, which are identical to those in Fig. 7, are shown in (a). Compared with Fig. 7, each resonance curve splits into two due to the interaction between the ungated resonators.

the ungated resonators. As a result, the gated resonances become hybridized and each of the corresponding resonance curves splits into two. This result follows from all models, as shown in Fig. 9. Around $590 \mathrm{GHz}$, the full-wave simulations agree better, as before, with the numerical and analytical solutions of the mode-matching equations than with the transmission-line model and the effective-medium model. The modified transmission-line model predicts much weaker interaction between gated resonators than the other models. We attribute this to higher values of $\left|R_{\mathrm{ug}}\right|$ yielded by this model (see Fig. 4).

In all the preceding examples, the geometry of the junctions between the gated and ungated sections remained the

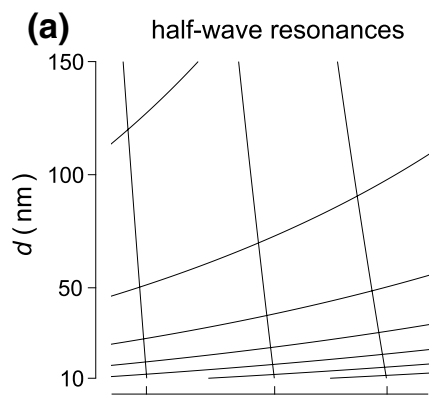

(b) effective medium

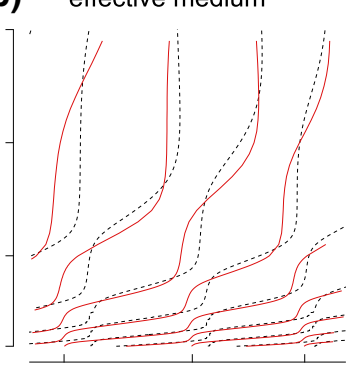

(c) modified transmission line

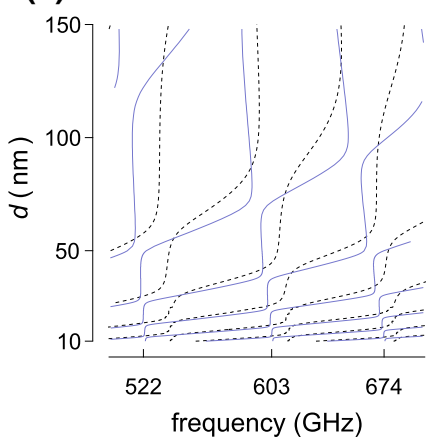

(d) analytical modal analysis

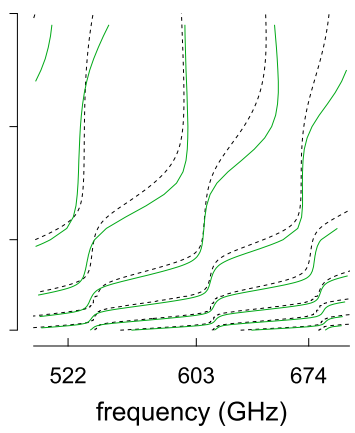

FIG. 10. Variation of the resonances for the three-section structure in Fig. 6 and for various junction geometries. The electron densities in the gated and ungated sections are $6.5 \times 10^{11} \mathrm{~cm}^{-2}$. Full-wave simulations are shown in (b)-(f) by dashed lines for comparison and as a guide for the eye. The half-wavelength resonances of the isolated sections are shown in (a). The best agreement is between analytical modal analysis and full-wave simulations.

same, even though the constitution of the sections and their electron density varied. We now discuss how plasmon resonances are affected by the junction geometry. The parameter responsible is $d$, which is both the gate-to-channel separation in the gated systems and the thickness of the dielectric above the channel in the ungated systems. We consider the three-section structure that has two ungated sections of lengths 20 and $3 \mu \mathrm{m}$ and a gated section of length $3 \mu \mathrm{m}$. We compare three analytical models - the effective-medium model, the modified transmission-line model, and analytical modal analysis - with full-wave simulations.

Figure 10(a) shows the half-wavelength resonances for the isolated sections, where the electron concentrations in the gated and ungated sections are $6.5 \times 10^{11} \mathrm{~cm}^{-2}$ and $d$ varies between 10 and $150 \mathrm{~nm}$. The gated and ungated resonant frequencies both depend on the value of $d$, in agreement with the dispersion relations for gated and ungated plasmons [Eqs. (10) and (11)]. Figures 10(b)-10(d) show the resonance curves of the whole structure. In each subfigure, the results of an analytical model are shown together with the results from the full-wave simulations (dashed 

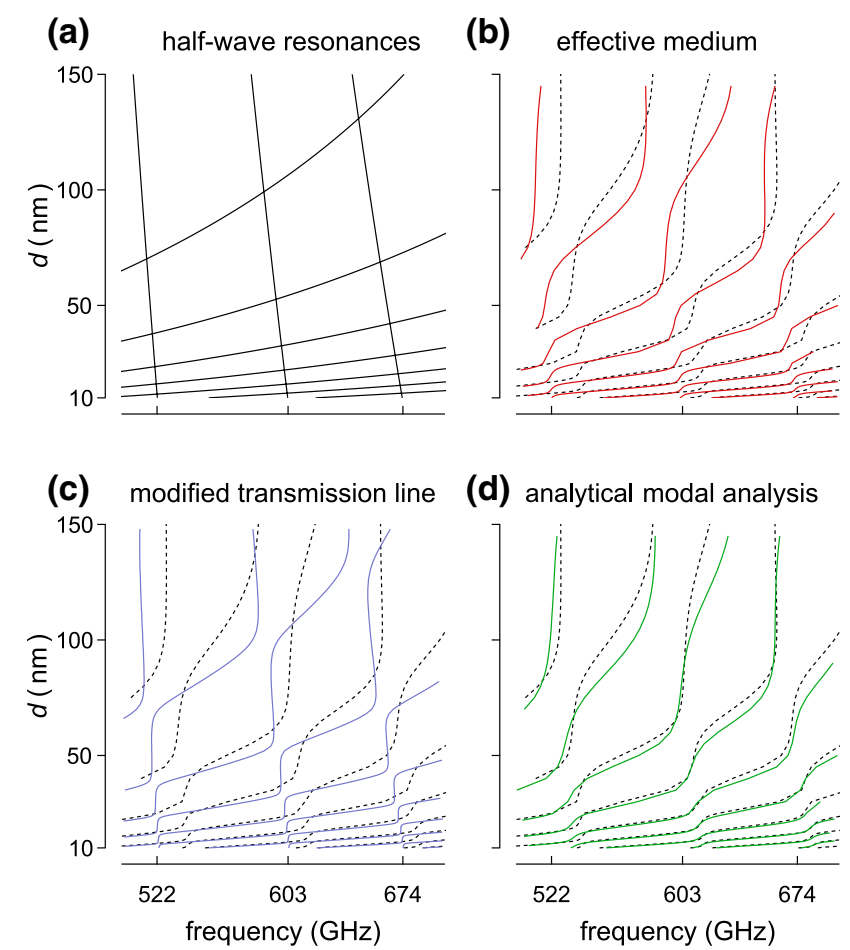

FIG. 11. The same as in Fig. 10 but with the electron density of the gated section equal to $4.9 \times 10^{11} \mathrm{~cm}^{-2}$. Full-wave simulations agree best with the analytical modal analysis.

lines). The curves obtained by all four methods show typical anticrossing behavior. However, the positions and the size of the anticrossing regions obtained by the effectivemedium and transmission-line models differ from those obtained by the full-wave simulations, leading to only qualitative agreement between the three approaches. The results of the analytical model analysis, on the other hand, agree much better with those of the full-wave simulations. The curves match closely for lower values of $d$ and frequency. The slight deterioration of the agreement for higher values of $d$ and the frequency is expected given the assumption of small $\kappa_{u(g)} d$ used in the analytical model. Figure 11 shows the same set of curves as in Fig. 10 but now for a gated electron density of $4.9 \times 10^{11} \mathrm{~cm}^{-2}$. As before, the curves obtained by the analytical modal analysis match closely those obtained by the full-wave simulations. The results obtained by the effective-medium and transmission line models fail to agree with those obtained by the full-wave simulations. We repeat the same calculations for a gated electron density of $3.1 \times 10^{11} \mathrm{~cm}^{-2}$ and see equally good quantitative agreement between the analytical modal analysis and the full-wave simulations.

The analysis in this section ignored plasmon loss. Radiation is negligible, and Ohmic contacts are perfect reflectors. Plasmon loss due to scattering can be incorporated in the model by addition of the term $v / \tau$ to the left-hand side of Euler's equation (1), where $\tau$ is a scattering time. In the resonant regime $\omega \tau \gg 1$, the presence of loss will not affect the resonant frequencies, and the quality factors of the resonators will be determined by the product $\omega \tau$.

\section{DISCUSSION AND CONCLUSIONS}

Both the effective-medium model and the transmissionline model are particularly convenient from the practical point of view. In the transmission-line model, the matrices of individual sections of a 2DES can be readily constructed, and whole systems can be described by a product of individual matrices. The effective-medium model gives simple analytical expressions for plasmon reflection and transmission coefficients.

The original formulation of the transmission-line model by Aizin and Dyer considered a 2DES embedded in infinite homogeneous dielectrics. However, unless the dielectric layer above the 2DES (see Fig. 1) is considerably thick compared with plasmon field localization, the effect of the air-dielectric interface cannot be ignored in practical devices. In our examples, the calculations by the original transmission-line model failed to agree with those by all other models. We, therefore, develop this model further and show that the air-dielectric interface affects the expressions for the ungated characteristic impedance and the plasmon power flow.

The lossless characteristic impedances in the transmission-line and effective-medium models are real valued, which leads to real-valued plasmon reflection and transmission coefficients. Our other models, however, produce complex-valued reflection and transmission coefficients. The difference between the models is greatest for the phase of the reflection coefficient $R_{\mathrm{ug}}$, which is close to $\pi / 4$ in our examples as found by mode-matching analysis and full-wave simulations. Consequently, for those values of frequency where ungated resonances dominate, we see quantitative disagreement between the transmission-line model and the effective-medium model, on the one hand, and the modal analysis and the full-wave simulations, on the other hand.

Solution of the modal equations relies on the orthogonality of the eigenmodes in the waveguides, which allows these equations to be reduced to integral-algebraic equations whose coefficients can be found analytically. This, in turn, reduces the computational burden of the subsequent numerical solution.

Furthermore, we use modal analysis to obtain approximate, but fully analytical, solutions for the practically significant case of a plasmon incident on a junction between a gated and an ungated 2DES (see Sec. III and Appendix B). This model, in contrast to other analytical models we considered, can yield nontrivial values of the phase of the plasmon reflection and transmission coefficients, which 
can be most readily appreciated from Eqs. (21a) and (21b). The nontrivial phase has a significant impact on the resonances of compound structures. The analytical model agrees well with numerical calculations but requires significantly less effort to implement. It could, therefore, be a useful and convenient tool for the analysis and design of experimental structures.

Modal analysis as well as full-wave simulations take into account the entire geometry of a junction. They do so by relying on Maxwell's equations to match the fields along the cross section of the junction, and not only on the 2DES. As a result, these models do not require additional boundary conditions. The agreement between these models, for ranges of frequencies, electron densities, and gate-to-channel separations, reinforces further the conclusion about the role that the entire junction geometry plays in transmission and reflection of 2D plasmons.

Full-wave simulations of 2DES structures can be established with a general-purpose numerical package, and they are free from the approximations of the other models. One such approximation is that individual gated and ungated resonators are coupled via transmission and reflection of plasmons only. However, as the mode-matching analysis shows, evanescent modes are also excited at the waveguide junctions. Although these modes decay away from the junctions, they may still affect the opposite side of a resonator if the resonator is sufficiently short. It is a possible cause of the discrepancy between the modal analysis and the full-wave simulations, the agreement between them being poorer for the structure containing short, $3-\mu \mathrm{m} \mathrm{sec-}$ tions (see Fig. 6) than for the structures containing longer, $10-\mu \mathrm{m}$ sections (see Figs. 7 and 9).

Finally, although our examples are for structures made in Ga-As/Al-Ga-As heterostuctures, the general analysis should be valid for other two-dimensional materials, provided they have a Drude conductivity.

The data presented in this paper are available at [44] and are used under the Creative Commons Attribution license.

\section{ACKNOWLEDGMENTS}

S.S. acknowledges financial support by Imperial College (President's Scholarship) and S.Z., J.E.C., and O.S. acknowledge financial support by the EPSRC (Grants No. EP/R004994/1 and No. EP/R00501X).

\section{APPENDIX A: TRANSMISSION-LINE MODEL IN THE PRESENCE OF THE DIELECTRIC-AIR INTERFACE}

The hydrodynamic and electrostatic approximations, after the standard linearization for the ac electron density and velocity, yield the following equations for a gated waveguide:

$$
\begin{array}{r}
\frac{\partial j(z, t)}{\partial t}=-\frac{e^{2} n_{0}}{m^{*}} \frac{\partial \phi(x=0, z, t)}{\partial z}, \\
\varepsilon_{0} \varepsilon q(1+\operatorname{coth} q d) \frac{\partial \phi(x=0, z, t)}{\partial t}=-\frac{\partial j(z, t)}{\partial z} .
\end{array}
$$

Equation (A1) are in the form of telegrapher's equations used to describe a transmission line. Substituting $L=$ $m^{*} / e^{2} n_{0}$ and $C_{g}=\varepsilon_{0} \varepsilon q(1+\operatorname{coth} q d)$ into the expressions in Eq. (A1) and defining $j(z, t)=I$ and $\phi(x=0, z, t)=V$ we obtain

$$
\begin{aligned}
L \frac{\partial I}{\partial t} & =-\frac{\partial V}{\partial z}, \\
C_{g} \frac{\partial V}{\partial t} & =-\frac{\partial I}{\partial z} .
\end{aligned}
$$

On the other hand, the telegrapher's equations for ungated waveguides take the form

$$
\begin{array}{r}
\frac{\partial j(z, t)}{\partial t}=-\frac{e^{2} n_{0}}{m^{*}} \frac{\partial \phi(x=0, z, t)}{\partial z}, \\
\frac{2 \varepsilon_{0} \varepsilon q}{(1+\Lambda)} \frac{\partial \phi(x=0, z, t)}{\partial t}=-\frac{\partial j(z, t)}{\partial z},
\end{array}
$$

where $\Lambda=(1-1 / \varepsilon) /(1+1 / \varepsilon) e^{-2 q d}$.

Using $C_{u}=2 \varepsilon_{0} \varepsilon q /(1+\Lambda)$, we can write the expressions in Eq. (A3) as

$$
\begin{aligned}
L \frac{\partial I}{\partial t} & =-\frac{\partial V}{\partial z}, \\
C_{u} \frac{\partial V}{\partial t} & =-\frac{\partial I}{\partial z} .
\end{aligned}
$$

Following Ref. [24], we impose the boundary conditions between gated and ungated sections on the basis of the continuity of the potential and the power flow through the boundary.

The power flow is defined as

$$
P(z)=\int_{-\infty}^{\infty} d x\left\langle S_{z}\right\rangle
$$

It can be shown [24] that

$$
P(z)=\frac{\phi(x=0, z, t) j^{*}(z, t)}{2}+\frac{i \varepsilon_{0} \varepsilon \omega}{2} \int_{-\infty}^{\infty} \phi \frac{\partial \phi^{*}}{\partial z} d x,
$$

where the asterisk denotes the complex conjugate. 
For a gated waveguide, Eq. (A6) yields

$$
P_{g}(z)=\frac{V I^{*}}{2}\left(1-\frac{1-e^{-2 q d}(1+2 q d)}{2\left(1-e^{-2 q d}\right)}\right) .
$$

For an ungated waveguide, Eq. (A6) yields

$$
P_{u}(z)=\frac{V I^{*}}{2}\left(1-\frac{1+[q d+(3 \varepsilon+1) / \varepsilon+1] \Lambda}{2(1+\Lambda)}\right),
$$

where $\lambda=(1-1 / \varepsilon) /(1+1 / \varepsilon)$.

The transmission matrix of a three-section system is given as a product of transmission matrices of individual sections, from which the eigenmode frequencies can be found.

\section{APPENDIX B: ANALYTICAL SOLUTIONS OF MODE-MATCHING EQUATIONS}

We first consider a plasmon incident on a junction between a gated and an ungated waveguide from the ungated waveguide. Assuming that the field at the junction is proportional to the field of the incident plasmon, $\mathcal{H}_{y}=C_{u} H_{u}$, and using the variational technique, we get the following equation for the variational parameter $C_{u}$ :

$$
C_{u}\left(k_{z, p}^{u} S_{p}^{u}+k_{z, p}^{g} \frac{J_{p p}^{2}}{S_{p}^{g}}+\int_{0}^{\infty} k_{z} \frac{J_{p t}^{2}}{S_{t}^{g}} d k_{x}^{e}+\int_{0}^{\infty} k_{z} \frac{J_{p b}^{2}}{S_{b}^{g}} d k_{x}^{e}\right)=2 .
$$

The transverse wave numbers of the gated and ungated plasmons both have imaginary values, and so we define $k_{x, p}^{u}=-i \kappa_{u}$ and $k_{x, p}^{g}=-i \kappa_{g}$, and $\kappa_{u(g)} \geq 0$. Next, we make several assumptions about the shape of the plasmon fields and about the localization of the plasmon energy. First, we take the terms $\kappa_{u} x$ and $\kappa_{g} x$ in the interval $x \in(0 ; d]$ as small parameters in power-series expansions [because of typical values of wave vectors for low terahertz frequencies (approximately $10^{6} \mathrm{rad} / \mathrm{m}$ ) and the depths of the 2DES (approximately 10-100 nm)]. The self-overlap integral $S_{p}^{u}$ is a sum of three terms:

$$
S_{p}^{u}=\int_{d}^{\infty} \Gamma_{u}\left(k_{z, p}^{u}\right)^{2} \frac{4 \zeta^{2} e^{-2 \kappa_{u} d}}{(1+\zeta)^{2}} e^{-2 i k_{x, p}^{u, a i r}(x-d)} d x+\frac{1}{\varepsilon}\left\{\int_{0}^{d}\left[\left(1-\Gamma_{u}\left(k_{z, p}^{u}\right)\right) e^{-\kappa_{u} x}-\Gamma_{u}\left(k_{z, p}^{u}\right) e^{-\kappa_{u} x}\right]^{2} d x+\int_{-\infty}^{0}\left(e^{\kappa_{u} x}\right)^{2} d x\right\}
$$

where $k_{x, p}^{u, \text { air }}=i \sqrt{\kappa_{u}^{2}+(\varepsilon-1) \omega^{2} / c^{2}}$ is a plasmon wave number in air and $\zeta=k_{x, p}^{u} / \varepsilon k_{x, p}^{u \text {,air }}=\kappa_{u} / \varepsilon \sqrt{\kappa_{u}^{2}+(\varepsilon-1)\left(\omega^{2} / c^{2}\right)}$. Performing integration in Eq. (B3) yields

$$
\begin{aligned}
S_{p}^{u}= & \Gamma_{u}\left(k_{z, p}^{u}\right)^{2} \frac{2 \zeta^{2} e^{-2 \kappa_{u} d}}{(1+\zeta)^{2}} \frac{1}{\left|k_{x, p}^{u, \text { air }}\right|} \\
& +\frac{1}{\varepsilon}\left[\left(1-\Gamma_{u}\left(k_{z, p}^{u}\right)\right)^{2} \frac{1-e^{-2 \kappa_{u} d}}{2 \kappa_{u}}-\Gamma_{u}\left(k_{z, p}^{u}\right)^{2} \frac{1-e^{2 \kappa_{u} d}}{2 \kappa_{u}}-2 \Gamma_{u}\left(k_{z, p}\right)\left(1-\Gamma_{u}\left(k_{z, p}^{u}\right)\right) d+\frac{1}{2 \kappa_{u}}\right] .
\end{aligned}
$$

Expanding this in a power series with respect to $\kappa_{u} d$ yields

$$
\begin{aligned}
S_{p}^{u} \approx & \Gamma_{u}\left(k_{z, p}^{u}\right)^{2} \frac{2 \zeta^{2}}{(1+\zeta)^{2}} \frac{1-2 \kappa_{u} d}{\left|k_{x, p}^{u, a i r}\right|} \\
& +\frac{1}{2 \kappa_{u} \varepsilon}\left\{1+2\left[1-2 \Gamma_{u}\left(k_{z, p}^{u}\right)\right]^{2} \kappa_{u} d\right\} .
\end{aligned}
$$

From the dispersion relation for an ungated plasmon [Eq. (11)], one gets

$$
1-2 \Gamma_{u}\left(k_{z, p}^{u}\right)=\Gamma_{u}\left(k_{z, p}^{u}\right)\left(\frac{1-\zeta}{1+\zeta} e^{-2 \kappa_{u} d}-1\right)
$$

assuming frequencies below $1 \mathrm{THz}$ and typical values $\Gamma_{u}\left(k_{z, p}^{u}\right) \sim \frac{1}{2}$.

Furthermore,

$$
\zeta=\frac{\kappa_{u}}{\varepsilon \sqrt{\kappa_{u}^{2}+(\varepsilon-1) \frac{\omega^{2}}{c^{2}}}}=\frac{1}{\varepsilon} \sqrt{\frac{1}{1+(\varepsilon-1) \frac{\omega^{2}}{\kappa_{u}^{2} c^{2}}}}
$$

But $\varepsilon=12.4$ and $\omega^{2} / c^{2} \ll \kappa_{u}^{2}$, and thus $\zeta \approx(1 / \varepsilon)$ $\left[1-(\varepsilon-1) \omega^{2} / \kappa_{u}^{2} c^{2}\right] \ll 1$. 
Thus,

$$
\begin{aligned}
\frac{2 \zeta^{2}}{(1+\zeta)^{2}} \approx 2 \zeta^{2}, \quad\left|k_{x, p}^{u, a i r}\right| & =\frac{\kappa_{u}}{\varepsilon \zeta} \approx \frac{\kappa_{u}}{1-\left[\varepsilon-1\left(\omega^{2} / \kappa_{u}^{2} c^{2}\right)\right]} \\
\frac{1-\zeta}{1+\zeta} e^{-2 \kappa_{u} d}-1 & \approx(1-2 \zeta)\left(1-2 \kappa_{u} d\right)-1 \\
& \approx-2\left(\zeta+2 \kappa_{u} d\right) .
\end{aligned}
$$

So

$$
\begin{aligned}
S_{p}^{u} & \approx 2 \Gamma_{u}\left(k_{z, p}^{u}\right)^{2} \zeta^{2} \frac{1-(\varepsilon-1)\left(\omega^{2} / \kappa_{u}^{2} c^{2}\right)}{\kappa_{u}}\left(1-2 \kappa_{u} d\right)+\frac{1}{2 \kappa_{u} \varepsilon}\left[1+8\left(\zeta+\kappa_{u} d\right)^{2} \kappa_{u} d\right] \\
& \approx \frac{1}{2 \varepsilon \kappa_{u}}\left[1+\frac{4 \Gamma_{u}\left(k_{z, p}^{u}\right)^{2}}{\varepsilon}\left(1-2 \kappa_{u} d\right)+8 \kappa_{u} d\left(\zeta+\kappa_{u} d\right)^{2}\right] \approx \frac{1}{2 \varepsilon \kappa_{u}} .
\end{aligned}
$$

The self-overlap integral of a plasmon in an ungated 2DES in a homogeneous dielectric is

$$
\tilde{S}_{p}^{u}=\frac{1}{\varepsilon}\left[\int_{0}^{\infty}\left(e^{-\tilde{\kappa_{u}} x}\right)^{2} d x+\int_{-\infty}^{0}\left(e^{\tilde{\kappa_{u}} x}\right)^{2} d x\right]=\frac{1}{\varepsilon \tilde{\kappa_{u}}} .
$$

It might appear that introducing the air-dielectric interface halves the plasmon energy. Careful consideration, however, shows that the energy remains almost the same (exactly the same within zeroth order of $\kappa_{u} d$ and $\zeta$ ).

The self-overlap integral $S_{p}^{g}$ for the gated plasmon is a sum of two terms corresponding to the space between the gate and the 2DES and in the dielectric under the 2DES, so

$$
\begin{aligned}
S_{p}^{g} & =\frac{1}{\varepsilon}\left[\int_{0}^{d}\left(\frac{\cosh \kappa_{g}(x-d)}{\sinh \kappa_{g} d}\right)^{2} d x+\int_{-\infty}^{0}\left(e^{-\kappa_{g} d}\right)^{2} d x\right] \\
& =\frac{1+\operatorname{coth} \kappa_{g} d}{2 \varepsilon \kappa_{g}}\left[1+\kappa_{g} d\left(\operatorname{coth} \kappa_{g} d-1\right)\right] .
\end{aligned}
$$

The overlap integral $J_{p p}$ comprises similarly two parts:

$$
J_{p p}=\frac{1}{\varepsilon}\left[\int_{0}^{d} \frac{\cosh \kappa_{g}(x-d)}{\sinh \kappa_{g} d} e^{-\kappa_{u} d} d x+\int_{-\infty}^{0} e^{\kappa_{g} d} e^{\kappa_{u} d} d x\right] .
$$

However, the terms $\kappa_{g} d, \kappa_{g} x$, and $\kappa_{u} x$ in the first integral are much smaller than unity and, therefore,

$$
\begin{aligned}
& \frac{\cosh \kappa_{g}(x-d)}{\sinh \kappa_{g} d} e^{-\kappa_{u} d} \\
& \approx \frac{\cosh \kappa_{g} x \cosh \kappa_{g} d-\sinh \kappa_{g} x \sinh \kappa_{g} d}{\sinh \kappa_{g} d}\left(1-\kappa_{u} d\right) \\
& \approx \frac{1}{\kappa_{g} d} .
\end{aligned}
$$

Therefore,

$$
\begin{aligned}
J_{p p} & =\frac{1}{\varepsilon}\left(\int_{0}^{d} \frac{1}{\kappa_{g} d} d x+\int_{-\infty}^{0} e^{\kappa_{g} d} e^{\kappa_{u} d} d x\right) \\
& =\frac{1}{\varepsilon}\left(\frac{1}{\kappa_{g}}+\frac{1}{\kappa_{u}+\kappa_{g}}\right) .
\end{aligned}
$$

We now turn to the third term in Eq. (B1). The fields of the evanescent modes below the 2DES can be written in the form $A\left(k_{x}^{e}\right) e^{i k_{x}^{e} x}+B\left(k_{x}^{e}\right) e^{-i k_{x}^{e} x}$, where

$$
\begin{aligned}
& A(k)=\Gamma_{g}(k) e^{i k d}+\left(1-\Gamma_{g}(k)\right) e^{-i k d}, \\
& B(k)=\left(1+\Gamma_{g}(k)\right) e^{i k d}+\Gamma_{g}(k) e^{-i k d} .
\end{aligned}
$$

The self-overlap integral $S_{b}^{g}$ is given by [33]

$$
\begin{aligned}
S_{b}^{g}= & 2 \pi A(k) B(k)=\frac{2 \pi}{\varepsilon}\left[1+2 \Gamma_{g}\left(k_{x}^{e}\right)^{2}\left(\cos 2 k_{x}^{e} d-1\right)\right. \\
& \left.+\Gamma_{g}\left(k_{x}^{e}\right) \sin 2 k_{x}^{e} d\right] .
\end{aligned}
$$

For $k_{x}^{e} \rightarrow \infty$, the envelope of $S_{b}^{g}\left(k_{x}^{e}\right)$ grows as $k^{2}$. 
The overlap integral $J_{p b}$ is found in a similar way as

$$
J_{p b}=\frac{1}{\varepsilon} \frac{A\left(k_{x}^{e}\right)\left(\kappa_{u}+i k_{x}^{e}\right)+B\left(k_{x}^{e}\right)\left(\kappa_{u}-i k_{x}^{e}\right)}{\kappa_{u}^{2}+\left(k_{x}^{e}\right)^{2}} .
$$

Using (B15a) and noting that $\Gamma_{g}(k) \propto k$, one can see that $J_{p b}^{2}(k \rightarrow \infty) \propto k^{-2}$. We can then write

$$
\int_{0}^{\infty} k_{z} \frac{J_{p b}^{2}}{S_{b}^{g}} d k_{x}^{e}=\int_{0}^{\infty} \sqrt{\varepsilon \frac{\omega^{2}}{c^{2}}-k^{2}} \frac{J_{p b}^{2}}{S_{b}^{g}} d k=\int_{0}^{\infty} J_{r}(k) d k .
$$

The envelope of the integrand behaves as $J_{r}(k \rightarrow \infty) \propto k^{-3}$ apart from the points of local minima of $S_{b}^{g}(k)$. These correspond to resonances between the gate and the 2DES. In the simplest case, these would satisfy an integer halfwavelength resonant condition $k d=\pi l, l \in \mathbb{N}$. This, however, means that plasmons will not couple effectively (because of a big difference between plasmon and resonant mode wave numbers) to such modes, and we can ignore these resonances.

Another interval where the value $S_{b}^{g}(k)$ is relatively small is near $k=0$. Indeed

$$
\begin{aligned}
S_{b}^{g}(k) & =\frac{2 \pi}{\varepsilon}\left[1+2 \Gamma_{g}(k)^{2}(\cos 2 k d-1)+\Gamma(k) \sin 2 k d\right] \\
& =\frac{2 \pi}{\varepsilon}\left[1+2 \Gamma_{g}(k)^{2}\left(-\frac{(2 k d)^{2}}{2}+\frac{(2 k d)^{4}}{4 !}-\cdots\right)+\Gamma(k) 2 k d-\frac{(2 k d)^{3}}{3 !}+\cdots\right] .
\end{aligned}
$$

Therefore, in the interval $k \in(0 ; 1 / 2 d), S_{b}^{g}(k)$ is relatively small to contribute significantly to the integral in Eq. (B18). On the other hand, for $k \geq k_{\mathrm{co}}=1 / 2 d$, the envelope of $S_{b}^{g}(k)$ has a large-enough value. Combining this with the assumption about the negligible impact of the resonances between the gate and the 2DES, we can replace the upper limit of integration in Eq. (B18) with $k=k_{\mathrm{co}}$.

In addition, it is possible now to expand the expressions for $S_{b}^{g}(k)$ and $J_{p b}\left(k, \kappa_{u}\right)$ into a power series using $k d$ and $\kappa_{u} d$ as small parameters.

Equation (B16) then yields

$$
S_{b}^{g}(k)=\frac{2 \pi}{\varepsilon} \frac{-2 k^{2} \gamma_{g}^{2}+\omega^{4}+2 k \gamma_{g}\left(k \gamma_{g} \cos 2 k d+i \omega \sin 2 k d\right)}{\omega^{4}} \approx \frac{2 \pi}{\varepsilon}\left(1-\frac{4 k^{4} \gamma_{g}^{2} d^{2}}{\omega^{4}}\right) .
$$

Similarly, Eq. (B17) yields

$$
J_{p b}(k)=\frac{2 \kappa_{u}\left[\omega^{2} e^{\kappa_{u} d}+2 i k\left(\gamma_{g}-\gamma_{u}\right) \sin k d-2 i \gamma_{u} \kappa_{u} \sinh \kappa_{u} d\right]}{\varepsilon \omega^{2}\left(\kappa_{u}^{2}+k^{2}\right)} \approx \frac{1}{\varepsilon} \frac{2 \kappa_{u}}{\kappa_{u}^{2}+k^{2}} .
$$

Therefore, Eq. (B18) takes the form

$$
\begin{aligned}
\int_{0}^{\infty} k_{z} \frac{J_{p b}^{2}}{S_{b}^{g}} d k_{x}^{e} & \approx \int_{0}^{k_{\mathrm{co}}} \sqrt{\varepsilon \frac{\omega^{2}}{c^{2}}-k^{2}} \frac{1}{2 \pi \varepsilon} \frac{4 \kappa_{u}^{2}}{\left(\kappa_{u}^{2}+k^{2}\right)^{2}\left(1-\frac{4 k^{4} \gamma_{g}^{2} d^{2}}{\omega^{4}}\right)} d k \\
& =\frac{2 \kappa_{u}^{2}}{\pi \varepsilon} \int_{0}^{k_{\mathrm{co}}} \sqrt{\varepsilon \frac{\omega^{2}}{c^{2}}-k^{2}} \frac{d k}{\left(\kappa_{u}^{2}+k^{2}\right)^{2}\left(1-\frac{4 k^{4} \gamma_{g}^{2} d^{2}}{\omega^{4}}\right)} .
\end{aligned}
$$


Now we can write $C_{u}$ explicitly as

$$
C_{u}=\frac{\varepsilon}{\frac{k_{z, p}^{u}}{4 \kappa_{u}}+\frac{\frac{k_{z, p}^{g}}{\kappa g}\left(1+\frac{\kappa g}{\kappa g+\kappa_{u}}\right)^{2}}{\left(1+\operatorname{coth} \kappa_{g} d\right)\left[1+\kappa_{g} d\left(\operatorname{coth} \kappa_{g} d-1\right)\right]}+\frac{\kappa_{u}^{2}}{\pi} \int_{0}^{k_{\mathrm{co}}} \sqrt{\varepsilon \frac{\omega^{2}}{c^{2}}-k^{2}} \frac{d k}{\left(\kappa_{u}^{2}+k^{2}\right)^{2}\left(1-\frac{4 k^{4} \gamma_{g}^{2} d^{2}}{\omega^{4}}\right)}} .
$$

While Eq. (B23) gives analytical expressions for $R_{\mathrm{ug}}, R_{\mathrm{gu}}, T_{\mathrm{ug}}$, and $T_{\mathrm{gu}}$ that significantly simplify numerical calculations if used, it appears too complicated in comparison with expressions such as those in Eq. (13).

It is possible to simplify Eq. (B23) even further if one ignores the higher-order terms in $J_{p p}$ and $S_{b}^{g}$, makes the electrostatic approximation $c \rightarrow \infty$, and takes the value of $\kappa_{u} / \kappa_{g}$ as a small parameter. The latter assumption can be shown to be valid for moderate values of $n_{g} / n_{u}$. The plasmon dispersion relations (10) and (11) yield

$$
\kappa_{u}=\frac{\omega^{2}}{2\left|\gamma_{u}\right|}, \quad \kappa_{g} d=\frac{1}{2} \frac{1}{1+\Gamma_{g}},
$$

so the value of $\kappa_{u} / \kappa_{g}=\left(n_{g} / n_{u}\right)\left[\kappa_{g} d /\left(1-2 \kappa_{g} d\right)\right]$ is small. The integral in Eq. (B18) can then be found as

$$
\int_{0}^{\infty} \frac{k_{z}}{k_{z, p}^{u}} \frac{J_{p b}^{2}}{S_{b}^{g} S_{p}^{u}} d k_{x}^{e} \approx-i \frac{2}{\pi},
$$

and the expression for the variational parameter takes the form

$$
C_{u}=\frac{2}{1+2 \kappa_{g} d-i \frac{2}{\pi}} .
$$

From here the expressions for the reflection and transmission coefficients Eqs. (19) and (20)] reduce to the form of the expressions in Eqs. (21a) and (21b).

[1] M. Dyakonov and M. Shur, Shallow Water Analogy for a Ballistic Field-Effect Transistor: New Mechanism of Plasma Wave Generation by a DC Current, Phys. Rev. Lett. 71, 2465 (1993).

[2] V. Ryzhii, A. Satou, and M. S. Shur, Plasma instability and terahertz generation in HEMTs due to electron transit-time effect, IEICE Trans. Electron. E89-C, 1012 (2006).

[3] T. Otsuji, Y. M. Meziani, M. Hanabe, T. Ishibashi, T. Uno, and E. Sano, Grating-bicoupled plasmon-resonant terahertz emitter fabricated with GaAs-based heterostructure material systems, Appl. Phys. Lett. 89, 263502 (2006).

[4] G. R. Aizin, J. Mikalopas, and M. Shur, Current-driven plasmonic boom instability in three-dimensional gated periodic ballistic nanostructures, Phys. Rev. B 93, 195315 (2016).

[5] O. Sydoruk, R. R. A. Syms, and L. Solymar, Amplifying mirrors for terahertz plasmons, J. Appl. Phys. 112, 104512 (2012).

[6] M. Dyakonov and M. Shur, Detection, mixing, and frequency multiplication of terahertz radiation by twodimensional electronic fluid, IEEE Trans. Electron. Dev. 43, 380 (1996).
[7] S. Rudin, G. Rupper, A. Gutin, and M. Shur, Theory and measurement of plasmonic terahertz detector response to large signals, J. Appl. Phys. 115, 064503 (2014).

[8] V. Ryzhii, A. Satou, T. Otsuji, and M. S. Shur, Plasma mechanisms of resonant terahertz detection in a twodimensional electron channel with split gates, J. Appl. Phys. 103, 014504 (2008).

[9] W. Knap, S. Rumyantsev, M. S. Vitiello, D. Coquillat, S. Blin, N. Dyakonova, M. Shur, F. Teppe, A. Tredicucci, and T. Nagatsuma, Nanometer size field effect transistors for terahertz detectors, Nanotech. 24, 214002 (2013).

[10] J. H. Davies, The Physics of Low-Dimensional Semiconductors: An Introduction (CUP, New York, NY, USA, 1998).

[11] L. Ju, B. Geng, J. Horng, C. Girit, M. Martin, Z. Hao, H. A. Bechtel, X. Liang, A. Zettl, Y. R. Shen, and F. Wang, Graphene plasmonics for tunable terahertz metamaterials, Nature Nanotech. 6, 630 (2011).

[12] G. C. Dyer, G. R. Aizin, S. Preu, N. Q. Vinh, S. J. Allen, J. L. Reno, and E. A. Shaner, Inducing an Incipient Terahertz Finite Plasmonic Crystal in Coupled Two Dimensional Plasmonic Cavities, Phys. Rev. Lett. 109, 126803 (2012).

[13] G. C. Dyer, G. R. Aizin, S. J. Allen, A. D. Grine, D. Bethke, J. L. Reno, and E. A. Shaner, Induced transparency by 
coupling of Tamm and defect states in tunable terahertz plasmonic crystals, Nat. Photonics 7, 925 (2013).

[14] V. M. Muravev, P. A. Gusikhin, I. V. Andreev, and I. V. Kukushkin, Novel Relativistic Plasma Excitations in a Gated Two-Dimensional Electron System, Phys. Rev. Lett. 114, 106805 (2015).

[15] J. Wu, A. S. Mayorov, C. D. Wood, D. Mistry, L. Li, W. Muchenje, M. C. Rosamond, L. Chen, E. H. Linfield, A. G. Davies, and J. E. Cunningham, Excitation, detection, and electrostatic manipulation of terahertz-frequency range plasmons in a two-dimensional electron system, Sci. Rep. 5, 15420 (2015).

[16] J. Wu, O. Sydoruk, A. S. Mayorov, C. D. Wood, D. Mistry, L. Li, E. H. Linfield, A. G. Davies, and J. E. Cunningham, Time-domain measurement of terahertz frequency magnetoplasmon resonances in a two-dimensional electron system by the direct injection of picosecond pulsed currents, Appl. Phys. Lett. 108, 091109 (2016).

[17] P. Kopyt, B. Salski, J. Marczewski, P. Zagrajek, and J. Lusakowski, Parasitic effects affecting responsivity of subThz radiation detector built of a MOSFET, J. Infrared Milli. Terahz. Waves 36, 1059 (2015).

[18] S. J. Allen, D. C. Tsui, and R. A. Logan, Observation of the Two-Dimensional Plasmon in Silicon Inversion Layers, Phys. Rev. Lett. 38, 980 (1977).

[19] S. A. Mikhailov, Plasma instability and amplification of electromagnetic waves in low-dimensional electron systems, Phys. Rev. B 58, 1517 (1998).

[20] T. Otsuji, M. Hanabe, T. Nishimura, and E. Sano, A gratingbicoupled plasma-wave photomixer with resonant-cavity enhanced structure, Opt. Express 14, 4815 (2006).

[21] G. C. Dyer, J. D. Crossno, G. R. Aizin, E. A. Shaner, M. C. Wanke, J. L. Reno, and S. J. Allen, A plasmonic terahertz detector with a monolithic hot electron bolometer, J. Phys. Condens. Matter 21, 195803 (2009).

[22] O. Sydoruk, J. B. Wu, A. Mayorov, C. D. Wood, D. K. Mistry, and J. E. Cunningham, Terahertz plasmons in coupled two-dimensional semiconductor resonators, Phys. Rev. B 92, 195304 (2015).

[23] A. S. Petrov, D. Svintsov, V. Ryzhii, and M. S. Shur, Amplified-reflection plasmon instabilities in gratinggate plasmonic crystals, Phys. Rev. B 95, 045405 (2017).

[24] G. R. Aizin and G. C. Dyer, Transmission line theory of collective plasma excitations in periodic two-dimensional electron systems: Finite plasmonic crystals and Tamm states, Phys. Rev. B 86, 235316 (2012).

[25] M. A. Khorrami, S. El-Ghazaly, H. Naseem, and S.Q. Yu, Global modeling of active terahertz plasmonic devices, EEE Trans. Terahertz Sci. Technol. 4, 101 (2014).

[26] D. Wang, W. Zhao, X. Gu, W. Chen, and W. Yin, Wideband modeling of graphene-based structures at different temperatures using hybrid FDTD method, IEEE Trans. Nanotech. 14, 250 (2015).
[27] H. Nasari and M. S. Abrishamian, Electrically tunable, plasmon resonance enhanced, terahertz third harmonic generation via graphene, RSC Adv. 6, 50190 (2016).

[28] Y. Koseki, V. Ryzhii, T. Otsuji, V. V. Popov, and A. Satou, Giant plasmon instability in a dual-grating-gate graphene field-effect transistor, Phys. Rev. B 93, 245408 (2016).

[29] V. V. Popov, O. V. Polischuk, and M. S. Shur, Resonant excitation of plasma oscillations in a partially gated two-dimensional electron layer, J. Appl. Phys. 98, 033510 (2005).

[30] V. V. Popov, G. M. Tsymbalov, and M. S. Shur, Plasma wave instability and amplification of terahertz radiation infield-effect-transistor arrays, J. Phys. Condens. Matter 20, 384208-1-6 (2008).

[31] O. Sydoruk, Amplification and generation of terahertz plasmons in gated two-dimensional channels: Modal analysis, J. Appl. Phys. 115, 204507-1-7 (2014).

[32] O. Sydoruk, Drifting plasmons in open two-dimensional channels: Modal analysis, J. Phys. D: Appl. Phys. 46, 135103-1-8 (2013).

[33] O. Sydoruk, K. Choonee, and G. Dyer, Transmission and reflection of terahertz plasmons in two-dimensional plasmonic devices, IEEE Trans. Terahertz Sci. Technol. 5, 486 (2015).

[34] O. Sydoruk, R. R. A. Syms, and L. Solymar, Distributed gain in plasmonic reflectors and its use for terahertz generation, Opt. Express 20, 19618 (2012).

[35] A. L. Fetter, Edgemagnetoplasmons in a bounded twodimensional electron fluid, Phys. Rev. B 32, 7676 (1985).

[36] V. A. Volkov and S. A. Mikhailov, Edge magnetoplasmons: Low frequency weakly damped excitations in inhomogeneous two-dimensional electron systems, JETP 67, 1639 (1988).

[37] B. Rejaei and A. Khavasi, Scattering of surface plasmons on graphene by a discontinuity in surface conductivity, J. Opt. 17, 075002 (2015).

[38] C. Paul, Analysis of Multiconductor Transmission Lines (Wiley-Interscience IEEE Press, Hoboken, NJ, 2008).

[39] A. Wexler, Solution of waveguide discontinuities by modal analysis, IEEE Trans. Microwave Theory Tech. 15, 508 (1967).

[40] T. Rozzi and M. Mongiardo, Open Electromagnetic Waveguides (IET, London, 1996).

[41] S. E. Kocabas, G. Veronis, D. A. B. Miller, and S. Fan, Modal analysis and coupling in metal-insulator-metal waveguides, Phys. Rev. B 79, 035120 (2009).

[42] R. E. Collin, Field Theory of Guided Waves (WileyInterscience-IEEE, New York, 1991).

[43] E. Wolf and M. Born, Principles of Optics: Electromagnetic Theory of Propagation, Interference and Diffraction of Light (CUP, Cambridge, 1999).

[44] S. Siaber, S. Zonetti, J. E. Cunningham, and O. Sydoruk, Data for: "Terahertz plasmon resonances in two-dimensional electron systems: modeling approaches," https://doi.org/10.5281/zenodo.2606664 (2019). 